\begin{abstract}
In this work, an effective macroscale description is derived for the growth of tissue on a porous scaffold. A multiphase model is employed to describe the tissue dynamics; linearisation to facilitate a multiple-scale homogenisation provides an effective macroscale description, which incorporates dependence on the microscale structure and dynamics. In particular, the resulting description admits both interstitial growth and active cell motion. This model comprises Darcy flow, and differential equations for the volume fraction of cells within the scaffold and the concentration of nutrient, required for growth. These are coupled to underlying Stokes-type cell problems that provide permeability tensors, with multiscale dependence incorporating active cell motion, with which to parameterise the macroscale flow. A subset of solutions are illustrated by numerical simulations.
\end{abstract}




\title{
A multiphase multiscale model for nutrient limited tissue growth
}

\author{
E.C. Holden, J. Collis, B.S. Brook \& R.D. O'Dea \\ CMMB, School of Mathematical Sciences, University of Nottingham, \\ University Park, Nottingham, UK
}

January 2018

\section{Introduction}

Tissue growth is a complex phenomenon, comprising a wide range of processes, and spanning multiple spatial and temporal scales. Over many decades, a range of mathematical models has been constructed to describe the growth of tissue in applications that include tissue engineering, wound healing and cancer treatment. In tissue engineering, cells, scaffolds and biologically active molecules are combined to create functional tissues for diverse uses such as organ replacement [71], toxicological research [25, 67] and cultured meat [7]. Mathematical models have been used to provide insight into a range of tissue engineering processes, including: the influence of the bioreactor (tissue culture device) on tissue morphology [57, 78]; to investigate mechanotransduction [55] and cell behaviour under different bioreactor flow rates [60]; and to optimise scaffold design [16]. In the case of wound healing, recently proposed models have sought to investigate how tissue stress and growth contribute to successful healing [8], that is of particular relevance to diabetic and geriatric patients [65]. A number of models have been developed to investigate the uncontrolled division and growth associated with cancer including avascular growth [76, 77], the processes of angiogenesis [58] and importantly, the delivery [35] and effectiveness of cancer drugs.

The above models are applicable at the tissue scale, and thereby do not incorporate explicitly dependence on the tissue microstructure, or otherwise employ detailed computational approaches. Recently, the method of multiscale homogenisation has been utilised in a number of biological models $[2,44,48,53,54,61,69,70]$ as it allows the incorporation of important microscale effects at the macroscale, without the high computational cost associated with a microscale level description of a 
macroscale sized domain. Multiscale homogenisation has a rich history of use in the study of porous flow $[5,13,30,37,39,66]$, including in the study of soil and reservoirs [33, 62,63]. These methods, discussed and compared in $[22,59]$, are used to derive suitable macroscale equations from an underlying microscale description, rather than stating them $a b$ initio. Microscale information, such as the geometry and dynamics, is thereby incorporated into the 'effective' equations describing macroscale behaviour. In order to use these techniques one is required to make the assumptions that the micro- and macro-length scales are well-separated and that there is local microscale periodicity.

More recently, these techniques have begun to be employed in the context of growing materials. In O'Dea et al. [54] a simple model of tissue growth within a porous scaffold, represented by nutrient-limited microscale accretion of a rigid solid structure, was considered. A macroscale description of growth and transport was obtained using a multiple-scales technique, to accommodate explicit dependence on microscale dynamics and structure. A similar analysis by Penta et al. [61] described accretion in a poroelastic setting, the underlying model therein being popularly used for the description of cartilage $[26,38,43,46]$. To permit analysis, the authors of $[54,61]$ (and other similar studies) exploit asymptotic restrictions on the underlying model, considering slow (quasi-static) growth and linearised deformation. In Collis et al. [17], such assumptions are relaxed to consider a macroscale representation of finite volumetric nutrient-limited growth of a hyperelastic solid, employing the Arbitrary-Lagrangian-Eulerian approach [12] to ameliorate the challenges involved in applying two-scale asymptotics to such a system.

The underlying description of growth, and tissue dynamics in these models $[17,54,61]$ is highly idealised, being restricted to accretion $[54,61]$ or simple volumetric growth of a solid body [17]. An alternative approach is to employ a multiphase, or mixture theory, description [23, 73], that provides a natural framework to accommodate the complexity associated with tissue growth dynamics and has therefore been widely used in the context of tumour growth $[14,21,31,45]$ and tissue engineering $[40,41,55]$; see also the review article [52]. Of particular relevance here is the study of Lemon et al. [42] in which tissue growth within a tissue engineering scaffold is described using a porous flow mixture theory model. The growing cell population is represented by a viscous fluid as in [45] but additionally accommodates cell-cell interactions via the specification of extra pressure, introduced by Breward et al. [10].

More recently these ideas, of mixture theory and multiscale homogenisation, were brought together in a tumour model investigating drug transport and response [18, 19]. At the microscale the tumour was modelled as a multiphase fluid mixture and an effective macroscale 
description, of flow, transport and mixture evolution, was obtained via multiscale homogenisation. In this work, the limit of large interphase drag was adopted, as in $[27,56]$, in which case each phase is subject to a common velocity and pressure. Whilst this reduction permitted the multiscale analysis that followed, the resulting description can be viewed as essentially equivalent to an accretion type model with growth occuring in a thin boundary layer at the tumour periphery.

In this work we address this deficiency, employing a two phase viscous fluid model of the kind presented in [42] to obtain an effective macroscale description of nutrient limited tissue growth on an artificial scaffold, in which we relax the assumption of large interphase drag so that active cell motion is permitted, caused by their tendency to aggregate or repel. The model therefore accommodates a more complex description of tissue dynamics, as well as permitting volumetric growth. A fully nonlinear analysis is complicated by the more complex mass-transfer features that we consider (see Collis et al. [17] for a discussion). Moreover, our aim here is to obtain a more tractable description that permits coupling between micro- and macro-scale processes. We therefore consider a linearisation of the model, which permits a more straightforward multiscale analysis. The macroscale model that we derive comprises a Darcy flow, a partial differential equation for the volume fraction of cells within the scaffold, and an advection-reaction equation for the nutrient concentration, coupled to the underlying microscale dynamics via suitable problems defined on a prototypical 'unit cell'. These so-called 'cell problems', determine microscale behaviour that is subsequently employed to specify effective coefficients in the macroscale description. Importantly, and in contrast to other similar studies, these unit cell problems are themselves parameterised by the macroscale dynamics, so that the micro- and macroscale descriptions are fully coupled.

Whilst the model is presented here in the context of tissue engineering it has the potential to be applied to other applications, in particular the development of biofilms, for example in the subsurface [20] or in the fouling of filters [6].

This article is organised as follows. The setup of the multiphase model at the microscale is described in section 2 for a periodic microscale cell, followed by its linearisation about a steady state and analysis of its linear stability. In section 3 multiscale homogenisation is used to derive an effective macroscale description, incorporating dependence on the microscale structure and dynamics. As is often the case in this kind of analysis, we are required to make further constitutive assumptions in order to obtain a closed macroscale model. In particular, we must specify the motion of the free tissue boundary at the microscale. To provide insight, we therefore consider in detail (in section 4) the travelling wave 
behaviour of the underlying multiphase model (in a similar manner to [41]) to thereby rationalise a constitutive statement for the boundary velocity in terms of the model parameters. We present illustrative numerical solutions in a particular limit case in section 5, indicating how microstructural information and cells' motile characteristics influence macroscale model behaviour. Finally, in section 6 we make some concluding remarks and suggest possible directions for future work.

\section{Model formulation}

We consider a model of broad relevance to tissue engineering applications where tissue growth occurs on a structured periodic scaffold (see Figure 1 for sample periodic cells), such as can be achieved through the use of 3D printing $[36,75,79]$. The characteristic lengthscales of the full extent of the scaffold and the porestructure, denoted $L$, and the $l$ respectively, are well separated and related by the dimensionless parameter $\varepsilon>0$, where

$$
\varepsilon=\frac{l}{L} \ll 1
$$

Henceforth we refer to the two lengthscales as the micro- and macro-scale.

We model the scaffold, $\Omega_{S}$, as a rigid solid. The tissue, $\Omega_{T}$, is modelled as a two phase mixture of cells and interstitial fluid which covers the scaffold, whilst the interstitial space, $\Omega_{I}$, contains only fluid. Henceforth, we refer to the interstitial fluid as water, for concision. Both cells and water are modelled as viscous fluids, described by Stokes equations, an approach taken by [45]. As the motion of the two fluids is very slow the inertial terms can be neglected; if they are retained, we find that in view of the scalings we adopt, they are, in any case, of lower order than we consider. The three domains that comprise the periodic cell are illustrated in Figure 1.

Increase in the cell volume fraction of the mixture depends on the concentration of some generic nutrient, as well as the availability of water. Tissue growth is represented by movement of the boundary $\Gamma$, between $\Omega_{T}$ and $\Omega_{I}$. This may occur as a consequence of nutrient limited phase transition, increasing the cell volume fraction (especially in the case of differences in phase density) or due to cell aggregation/repulsion.

\subsection{Model equations}

\subsubsection{The tissue, $\Omega_{T}$}

We describe the tissue using a multiphase mixture approach. This has been used by many authors in the context of tissue growth, building on the general theory of multiphase (porous) flow developed in, for example, 


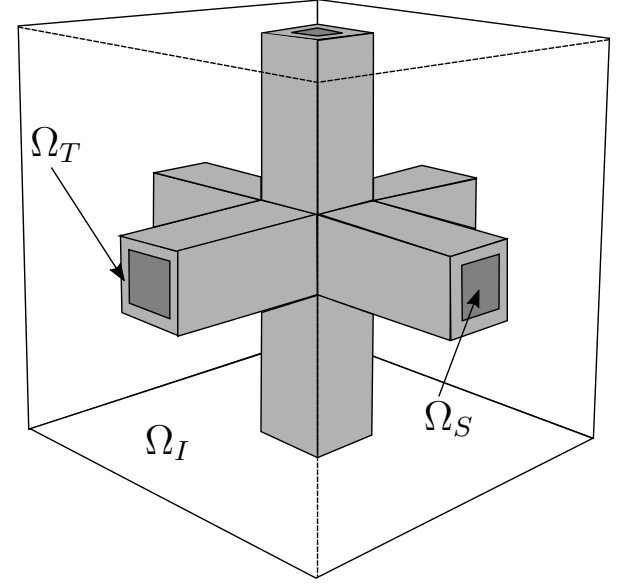

(a) Three-dimensional geometry

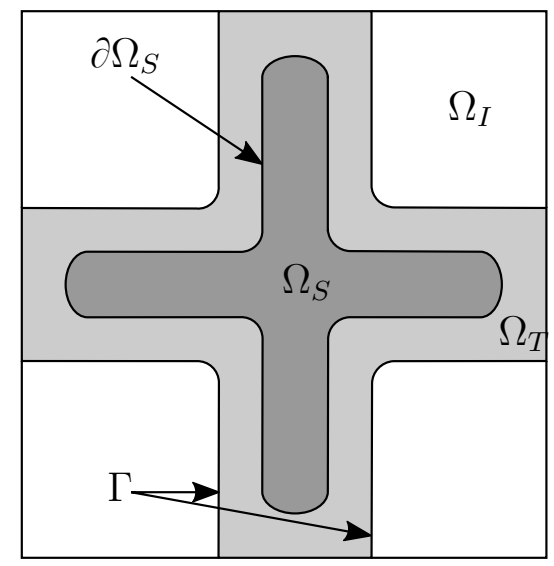

(b) Two-dimensional geometry

Figure 1: Schematic diagrams of the microscale domain, $\Omega$, composed of scaffold, $\Omega_{S}$, tissue $\Omega_{T}$ and interstitial fluid, $\Omega_{I}$. The scaffold boundary is denoted by $\partial \Omega_{S}$ and tissue-interstitial boundary by $\Gamma$. (a) A sample three-dimensional geometry. (b) A two-dimensional geometry used in the illustrative numerical experiments, section 5. We highlight that this geometry results in a disconnected interstitium, but we restrict attention to this simplified set-up for computational ease and thereby concentrate on the dynamics within $\Omega_{T}$ in our illustrative results.

$[9,24,47]$. We choose to follow closely [42], in which a mixture theory model for tissue growth in a porous scaffold is presented in general terms and for an arbitrary number of phases, including a widely applicable form for the mechanical interactions within and between phases.

Here, in our model, the tissue comprises two phases, cells and water, of volume fraction $\theta_{n}$ and $\theta_{w}$ respectively, satisfying the no voids constraint

$$
\theta_{n}+\theta_{w}=1
$$

The conservation of mass for each phase is given by

$$
\rho_{i}\left(\frac{\partial \theta_{i}}{\partial t}+\nabla \cdot\left(\theta_{i} \boldsymbol{v}_{i}\right)\right)=S_{i} \quad \text { for } i=n, w,
$$

where $\rho_{i}, \boldsymbol{v}_{i}$ and $S_{i}$ denote the phase densities, velocities and source terms, respectively. In order that mass is conserved we set $S_{n}=-S_{w}$. For generality we do not specify $S_{n}$ here but note that in general it will have functional dependence on $\theta_{n}, \theta_{w}$ and nutrient concentration $c$. A suitable function will be defined for the numerical experiments in section 5. Combining mass conservation (2.3) for each phase we obtain

$$
\boldsymbol{\nabla} \cdot\left(\theta_{n} \boldsymbol{v}_{n}+\theta_{w} \boldsymbol{v}_{w}\right)=\left(\frac{1}{\rho_{n}}-\frac{1}{\rho_{w}}\right) S_{n}
$$


The momentum equations are given by

$$
\boldsymbol{\nabla} \cdot\left(\theta_{i} \boldsymbol{\sigma}_{i}\right)+\boldsymbol{f}_{i j}=\mathbf{0} \quad \text { for } i=n, w,
$$

where the stress tensors, $\boldsymbol{\sigma}_{i}$, and interphase forces, $\boldsymbol{f}_{i j}$, are

$$
\begin{aligned}
& \boldsymbol{\sigma}_{i}=-p_{i} \boldsymbol{I}+\mu_{i}\left(\boldsymbol{\nabla} \boldsymbol{v}_{i}+\left(\boldsymbol{\nabla} \boldsymbol{v}_{i}\right)^{\top}-\frac{2}{3}\left(\boldsymbol{\nabla} \cdot \boldsymbol{v}_{i}\right) \boldsymbol{I}\right) \\
& \boldsymbol{f}_{i j}=p \boldsymbol{\nabla} \theta_{i}+\beta_{i j} \theta_{i} \theta_{j}\left(\boldsymbol{v}_{j}-\boldsymbol{v}_{i}\right) .
\end{aligned}
$$

In (2.6) $p_{i}$ denotes the pressure of the $i$ th phase, whose constant viscosity is $\mu_{i}$. In (2.7) we have assumed that the only interphase interaction that exists is passive viscous drag, with coefficient $\beta_{i j}$ (which we further set to be $\left.\beta_{n w}=\beta_{w n}=\beta\right) ; p$ denotes a common mixture pressure related to the individual phase pressures by $p_{w}=p$ and $p_{n}=p+\phi_{n}$. Active cell motion due to cell-cell interactions is accommodated via the additional pressure term $\phi_{n}$, here chosen (as in [42]) as follows:

$$
\phi_{n}=\theta_{n}\left(-\nu+\frac{\kappa \theta_{n}}{1-\theta_{n}}\right),
$$

We remark that the accommodation of cell-cell interactions (and active forces more generally) within a multiphase framework through the specification of extra pressure contributions has been widely employed (see for example, $[10,14,15,31,42])$. While the precise functional form of these contributions differs somewhat between authors, the key features are: (i) at low density, cells are expected to aggregate; (ii) this aggregation is curtailed at high density due to repulsive forces that exist between cells at high density. Here, the first, negative, term in (2.8) represents aggregation with strength $\nu>0$, while the second term curtails this, with strength $\kappa>0$. Note in particular the singularity that occurs as $\theta_{n}=1$, representing high repulsion occurring when all available space is occupied by cells.

In summary, the momentum equations are given by

$$
\begin{aligned}
\theta_{n} \boldsymbol{\nabla} p_{w}+ & \boldsymbol{\nabla}\left(\theta_{n} \phi_{n}\right)+\beta \theta_{n} \theta_{w}\left(\boldsymbol{v}_{n}-\boldsymbol{v}_{w}\right) \\
& -\boldsymbol{\nabla} \cdot\left(\mu_{n} \theta_{n}\left(\boldsymbol{\nabla} \boldsymbol{v}_{n}+\left(\boldsymbol{\nabla} \boldsymbol{v}_{n}\right)^{\top}-\frac{2}{3}\left(\boldsymbol{\nabla} \cdot \boldsymbol{v}_{n}\right) \boldsymbol{I}\right)\right)=\mathbf{0} \\
\theta_{w} \boldsymbol{\nabla} p_{w}+ & \beta \theta_{n} \theta_{w}\left(\boldsymbol{v}_{w}-\boldsymbol{v}_{n}\right) \\
& -\boldsymbol{\nabla} \cdot\left(\mu_{w} \theta_{w}\left(\boldsymbol{\nabla} \boldsymbol{v}_{w}+\left(\boldsymbol{\nabla} \boldsymbol{v}_{w}\right)^{\top}-\frac{2}{3}\left(\boldsymbol{\nabla} \cdot \boldsymbol{v}_{w}\right) \boldsymbol{I}\right)\right)=\mathbf{0}
\end{aligned}
$$


It is at times notationally convenient to refer to weighted mixture variables such as velocity and stress; these are represented by subscript $T$. For example, we define

$$
\begin{aligned}
& \boldsymbol{v}_{T}=\theta_{n} \boldsymbol{v}_{n}+\theta_{w} \boldsymbol{v}_{w}, \\
& \boldsymbol{\sigma}_{T}=\theta_{n} \boldsymbol{\sigma}_{n}+\theta_{w} \boldsymbol{\sigma}_{w} .
\end{aligned}
$$

\subsubsection{The interstitium, $\Omega_{I}$}

In this domain there are no cells, so $\theta_{n}=0, \theta_{w}=1$ and the flow is described by a Stokes flow:

$$
\begin{aligned}
& \boldsymbol{\nabla} \cdot \boldsymbol{v}_{I}=0 \\
& \boldsymbol{\nabla} p_{I}-\boldsymbol{\nabla} \cdot\left(\mu_{w}\left(\boldsymbol{\nabla} \boldsymbol{v}_{I}+\left(\boldsymbol{\nabla} \boldsymbol{v}_{I}\right)^{\top}\right)\right)=0
\end{aligned}
$$

where subscript $I$ denotes variables in $\Omega_{I}$.

\subsubsection{Nutrient}

A generic nutrient of concentration $c$, on which mitosis depends, is advected by the flows, diffuses with constant diffusivity $D_{i}$, and in $\Omega_{T}$ is taken up by the cell phase according to some function $\Lambda\left(\theta_{n}, c_{T}\right)$.

$$
\begin{aligned}
& \frac{\partial c_{I}}{\partial t}+\nabla \cdot\left(c_{I} \boldsymbol{v}_{I}\right)=\boldsymbol{\nabla} \cdot\left(D_{I} \boldsymbol{\nabla} c_{I}\right) \quad \text { in } \Omega_{I}, \\
& \frac{\partial c_{T}}{\partial t}+\boldsymbol{\nabla} \cdot\left(c_{T} \boldsymbol{v}_{T}\right)=\boldsymbol{\nabla} \cdot\left(D_{T} \boldsymbol{\nabla} c_{T}\right)-\Lambda \quad \text { in } \Omega_{T} .
\end{aligned}
$$

In the tissue, as in Lemon and King [40], we have assumed that the concentration of nutrient in the cell and water phases is equal, due to permeable cell membranes and reaching equilibrium rapidly on the microscale of the mixture. The sum of the nutrient transport equation in each phase results in (2.16), with the nutrient advected by the composite mixture velocity $(2.11)$ and $D_{T}$ representing the effective diffusion coefficient of the nutrient in the mixture.

We suppose that $D_{I}$ is constant but note that diffusivity in the tissue will, in general, have dependence on the cell volume fraction, i.e. $D_{T}=$ $D_{T}\left(\theta_{n}\right)$. However, in the linearisation that follows (Section 2.3) we find that this variation is relegated to lower order and so we do not specify it here. Additionally, and in the interest of generality, we defer specification of the uptake function $\Lambda$ to section 5 when we provide illustrative numerical experiments of a particular limit case. 


\subsubsection{Boundary conditions}

At the scaffold-tissue boundary we impose no slip and no penetration conditions so that

$$
\boldsymbol{v}_{i}=\mathbf{0} \text { on } \partial \Omega_{S},
$$

and since we assume that nutrient cannot enter the scaffold,

$$
\boldsymbol{\nabla} c_{T} \cdot \boldsymbol{n}_{S}=0 \text { on } \partial \Omega_{S}
$$

where $\boldsymbol{n}_{S}$ is the outward normal to the scaffold.

The tissue-interstitium boundary, $\Gamma$, exists along the surface where cells are present on one side but not on the other. Its position is given by

$$
\frac{\partial F}{\partial t}+\nabla F \cdot \boldsymbol{v}_{\Gamma}=0
$$

where $\boldsymbol{v}_{\Gamma}$ is the boundary velocity and the level set equation $F(\boldsymbol{x}, t)=0$ describes the position of the moving interface $\Gamma$.

On this boundary we impose the following flux conditions:

$$
\begin{aligned}
& \rho_{n} \theta_{n}\left(\boldsymbol{v}_{n}-\boldsymbol{v}_{\Gamma}\right) \cdot \boldsymbol{n}=0, \\
& \rho_{w} \theta_{w}\left(\boldsymbol{v}_{w}-\boldsymbol{v}_{\Gamma}\right) \cdot \boldsymbol{n}=\rho_{w}\left(\boldsymbol{v}_{I}-\boldsymbol{v}_{\Gamma}\right) \cdot \boldsymbol{n}, \\
& {\left[c_{i}\left(\boldsymbol{v}_{i}-\boldsymbol{v}_{\Gamma}\right) \cdot \boldsymbol{n}-D_{i} \boldsymbol{\nabla} c_{i} \cdot \boldsymbol{n}\right]_{-}^{+}=0 \text { where } i=T, I,}
\end{aligned}
$$

where $\boldsymbol{n}$ is the outward normal to the interface, $\Gamma$ and []$_{-}^{+}$denotes the jump across the boundary. The first of these simply implies that the normal velocity of the boundary is given by the normal velocity of the cells there; i.e. no cells are permitted in $\Omega_{I}$. The second enforces the conservation of mass flux of water across the boundary whilst the third describes the flux of nutrient concentration. In addition, we impose continuity of tangential velocity, normal stress and nutrient concentration on $\Gamma$ :

$$
\left[\boldsymbol{v}_{i} \cdot \boldsymbol{t}\right]_{-}^{+}=0 \quad\left[\boldsymbol{\sigma}_{i} \cdot \boldsymbol{n}\right]_{-}^{+}=\mathbf{0}, \quad\left[c_{i}\right]_{-}^{+}=0 \quad \text { for } i=T, I .
$$

\subsection{Nondimensionalisation}

We non-dimensionalise our model equations by using the following scalings:

$$
\begin{aligned}
& \boldsymbol{x}=l \hat{\boldsymbol{x}}, \quad \boldsymbol{v}=V \hat{\boldsymbol{v}}, \quad c=C \hat{c}, \quad p=\frac{\mu_{n} V}{l} \hat{p}, \quad t=\frac{l}{V} \hat{t}, \\
& S_{n}=\frac{\rho_{n} V}{l} \hat{S}_{n}, \quad \beta=\frac{\mu_{n}}{l^{2}} \hat{\beta}, \quad \mu=\frac{\mu_{w}}{\mu_{n}}, \quad \frac{D_{i}}{V l}=\frac{1}{P e_{i}}, \quad \Lambda=\frac{V}{l} \hat{\Lambda}, \quad \bar{\rho}=\frac{\rho_{n}}{\rho_{w}} .
\end{aligned}
$$

in which circumflexes denote dimensionless variables and $V$, and $C$ are a characteristic microscale velocity and nutrient concentration. These characteristic quantities are to be determined by the specific application, 
dependent, for example, on the imposed fluid pressure drop in the bioreactor and the choice of nutrient. In the interest of generality we do not specify them here. As we work exclusively in dimensionless variables in subsequent sections, we drop the circumflex notation for convenience.

\subsection{Steady state and linearisation}

As is typical in homogenisation problems of this type, where flow, transport and (more recently) growth of porous media is considered, we reduce the degree of non-linearity of the microscale model to enable a more straightforward multiscale analysis that follows; fully nonlinear approaches, such as that undertaken in Collis et al. [17] are discussed in section 6 . In Collis et al. [19] this was achieved through imposing large interphase drag, forcing the phase velocities within the mixture to be equal, and thereby removing the nonlinearities present in the continuity equations. To extend this model by allowing active cell motion, and hence distinct phase velocities, here we instead linearise the equations about a uniform steady state, across $\Omega_{T}$, with the following expansions:

$$
\begin{aligned}
\theta_{n} & =\theta_{n}^{*}+\delta \theta_{n, 1}+\ldots \\
\boldsymbol{v}_{i} & =\delta \boldsymbol{v}_{i, 1}+\ldots \\
p_{i} & =p_{i}^{*}+\delta p_{i, 1}+\ldots \\
c & =c^{*}+\delta c_{1}+\ldots \\
F & =F^{*}+\delta F_{1}+\ldots
\end{aligned}
$$

where $0<\delta \ll 1$ and asterisks denote steady state values. The steady state volume fraction and nutrient concentration are defined by

$$
S_{n}\left(\theta_{n}^{*}, \theta_{w}^{*}, c^{*}\right)=0, \quad \Lambda\left(\theta_{n}^{*}, c^{*}\right)=0,
$$

while the steady state velocity is $\boldsymbol{v}_{i}^{*}=\mathbf{0}$ and $p_{i}^{*}$ is an arbitrary constant. Whilst a steady state may exist within $\Omega_{T}$ that has $\boldsymbol{x}$-dependence, or one that varies on the macroscale, we do not consider this here for simplicity. We remark, however, that under the scalings that we employ in the subsequent multiscale analysis, the leading order cell volume fraction, nutrient concentration and pressure are uniform at the microscale, in partial support of our simplifying assumption.

The linearised equations are given below. In $\Omega_{T}$ :

$$
\begin{aligned}
\frac{\partial \theta_{n, 1}}{\partial t}+\theta_{n}^{*} \boldsymbol{\nabla} \cdot \boldsymbol{v}_{n, 1} & =S_{n, 1}, \\
\frac{\partial \theta_{w, 1}}{\partial t}+\theta_{w}^{*} \boldsymbol{\nabla} \cdot \boldsymbol{v}_{w, 1} & =-\bar{\rho} S_{n, 1}, \\
\theta_{n, 1}+\theta_{w, 1} & =0, \\
\nabla \cdot\left(\theta_{n}^{*} \boldsymbol{v}_{n, 1}+\theta_{w}^{*} \boldsymbol{v}_{w, 1}\right) & =(1-\bar{\rho}) S_{n, 1},
\end{aligned}
$$




$$
\begin{gathered}
\theta_{n}^{*}\left[\boldsymbol{\nabla}\left(p_{w, 1}+\phi_{n, 1}\right)+\beta \theta_{w}^{*}\left(\boldsymbol{v}_{n, 1}-\boldsymbol{v}_{w, 1}\right)-\nabla^{2} \boldsymbol{v}_{n, 1}-\frac{1}{3} \boldsymbol{\nabla}\left(\boldsymbol{\nabla} \cdot \boldsymbol{v}_{n, 1}\right)\right]=\mathbf{0} \\
\theta_{w}^{*}\left[\boldsymbol{\nabla} p_{w, 1}+\beta \theta_{n}^{*}\left(\boldsymbol{v}_{w, 1}-\boldsymbol{v}_{n, 1}\right)-\mu\left(\nabla^{2} \boldsymbol{v}_{w, 1}+\frac{1}{3} \boldsymbol{\nabla}\left(\boldsymbol{\nabla} \cdot \boldsymbol{v}_{w, 1}\right)\right)\right]=\mathbf{0} \\
\frac{\partial c_{T, 1}}{\partial t}+\boldsymbol{\nabla} \cdot\left(c^{*}\left(\theta_{n}^{*} \boldsymbol{v}_{n, 1}+\theta_{w}^{*} \boldsymbol{v}_{w, 1}\right)\right)=\frac{1}{P e_{T}} \nabla^{2} c_{T, 1}-\Lambda_{1} .
\end{gathered}
$$

where

$$
\begin{aligned}
S_{n, 1} & =\frac{\partial S_{n}}{\partial \theta_{n}}\left(\theta_{n}^{*}, c^{*}\right) \theta_{n, 1}+\frac{\partial S_{n}}{\partial c}\left(\theta_{n}^{*}, c^{*}\right) c_{T, 1}, \quad \phi_{n, 1}=\frac{1}{\theta_{n}^{*}} \frac{\partial\left(\theta_{n} \phi_{n}\right)}{\partial \theta_{n}}\left(\theta_{n}^{*}\right) \theta_{n, 1}, \\
\Lambda_{1} & =\frac{\partial \Lambda}{\partial \theta_{n}}\left(\theta_{n}^{*}, c^{*}\right) \theta_{n, 1}+\frac{\partial \Lambda}{\partial c}\left(\theta_{n}^{*}, c^{*}\right) c_{T, 1},
\end{aligned}
$$

are the linearised growth, uptake and intraphase pressure functions, respectively. Note that due to the linearisation, the Péclet number in the tissue domain is now a function of the steady state cell volume fraction $\theta_{n}^{*}$, and hence takes a constant value.

In $\Omega_{I}$ the flow is governed by:

$$
\begin{aligned}
\boldsymbol{\nabla} \cdot \boldsymbol{v}_{I, 1} & =0 \\
\boldsymbol{\nabla} p_{I, 1}-\mu \nabla^{2} \boldsymbol{v}_{I, 1} & =\mathbf{0} \\
\frac{\partial c_{I, 1}}{\partial t}+\boldsymbol{\nabla} \cdot\left(c^{*} \boldsymbol{v}_{I, 1}\right) & =\frac{1}{P e_{I}} \nabla^{2} c_{I, 1} .
\end{aligned}
$$

Boundary conditions are obtained by linearising $(2.17,2.18)$ on the scaffold surface, $\partial \Omega_{S}$ :

$$
\boldsymbol{v}_{n, 1}=\boldsymbol{v}_{w, 1}=\mathbf{0}, \quad \boldsymbol{\nabla} c_{T, 1} \cdot \boldsymbol{n}_{S}=0,
$$

while on the tissue boundary $\Gamma$ :

$$
\begin{gathered}
\frac{\partial F_{1}}{\partial t}+\boldsymbol{\nabla} F^{*} \cdot \boldsymbol{v}_{\Gamma, 1}=0, \\
\theta_{n}^{*}\left(\boldsymbol{v}_{n, 1}-\boldsymbol{v}_{\Gamma, 1}\right) \cdot \boldsymbol{n}=0, \\
\theta_{w}^{*}\left(\boldsymbol{v}_{w, 1}-\boldsymbol{v}_{\Gamma, 1}\right) \cdot \boldsymbol{n}=\left(\boldsymbol{v}_{I, 1}-\boldsymbol{v}_{\Gamma, 1}\right) \cdot \boldsymbol{n}, \\
-\left(p_{w, 1}+\theta_{n}^{*} \phi_{n, 1}\right) \boldsymbol{I}+\theta_{n}^{*}\left(\boldsymbol{\nabla} \boldsymbol{v}_{n, 1}+\left(\boldsymbol{\nabla} \boldsymbol{v}_{n, 1}\right)^{\top}-\frac{2}{3}\left(\boldsymbol{\nabla} \cdot \boldsymbol{v}_{n, 1}\right) \boldsymbol{I}\right) \\
+\mu \theta_{w}^{*}\left(\boldsymbol{\nabla} \boldsymbol{v}_{w, 1}+\left(\boldsymbol{\nabla} \boldsymbol{v}_{w, 1}\right)^{\top}-\frac{2}{3}\left(\boldsymbol{\nabla} \cdot \boldsymbol{v}_{w, 1}\right) \boldsymbol{I}\right) \\
=-p_{I, 1} \boldsymbol{I}+\mu\left(\boldsymbol{\nabla} \boldsymbol{v}_{I, 1}+\boldsymbol{\nabla} \boldsymbol{v}_{I, 1}^{\top}\right),
\end{gathered}
$$




$$
\begin{array}{r}
{\left[\boldsymbol{v}_{i, 1} \cdot \boldsymbol{t}\right]_{-}^{+}=0} \\
{\left[c^{*}\left(\boldsymbol{v}_{i, 1}-\boldsymbol{v}_{\Gamma, 1}\right) \cdot \boldsymbol{n}-\frac{1}{P e_{i}} \boldsymbol{\nabla} c_{i, 1} \cdot \boldsymbol{n}\right]_{-}^{+}=0,} \\
{\left[c_{i, 1}\right]_{-}^{+}=0}
\end{array}
$$

where $i=T, I$, and the linearised weighted mixture velocity is given by

$$
\boldsymbol{v}_{T, 1}=\theta_{n}^{*} \boldsymbol{v}_{n, 1}+\theta_{w}^{*} \boldsymbol{v}_{w, 1}
$$

\subsubsection{Stability}

In section 4 we investigate the travelling wave behaviour of the above model, in a simplified limit case, in order to rationalise a constitutive statement for the velocity of the free boundary $\Gamma$, as required to close the macroscale description derived in section 3. For general interest, and because of its importance in determining the travelling wave behaviour of section 4 , we next consider the stability of the steady state using the linearised equations in one dimension. We highlight that the analysis of the full model is presented here, while the results that pertain to a particular limit case are employed in section 4.

By assuming that the phase densities are equal, and by elimination of $\theta_{w}^{*}, \boldsymbol{v}_{w, 1}$ and $p_{w, 1}$, the equations in the tissue domain, $\Omega_{T}$, are reduced to

$$
\begin{aligned}
& \frac{\partial \theta_{n, 1}}{\partial t}+\theta_{n}^{*} \frac{\partial v_{n, 1}}{\partial x}=\frac{\partial S_{n}}{\partial \theta_{n}}\left(\theta_{n}^{*}, c^{*}\right) \theta_{n, 1}+\frac{\partial S_{n}}{\partial c}\left(\theta_{n}^{*}, c^{*}\right) c_{T, 1}, \\
& J\left(\theta_{n}^{*}\right) \frac{\partial \theta_{n, 1}}{\partial x}+\beta \frac{1}{1-\theta_{n}^{*}} v_{n, 1}-\frac{4}{3}\left(1+\mu \frac{\theta_{n}^{*}}{1-\theta_{n}^{*}}\right) \frac{\partial^{2} v_{n, 1}}{\partial x^{2}}=0, \\
& \frac{\partial c_{T, 1}}{\partial t}=\frac{1}{P e_{T} i} \frac{\partial^{2} c_{T, 1}}{\partial x^{2}}-\frac{\partial \Lambda}{\partial \theta_{n}}\left(\theta_{n}^{*}, c^{*}\right) \theta_{n, 1}-\frac{\partial \Lambda}{\partial c}\left(\theta_{n}^{*}, c^{*}\right) c_{T, 1},
\end{aligned}
$$

where

$$
J\left(\theta_{n}^{*}\right)=-2 \nu+\frac{\kappa \theta_{n}^{*}\left(3-2 \theta_{n}^{*}\right)}{\left(1-\theta_{n}^{*}\right)^{2}} .
$$

We remark that the formulation $(2.33-2.35)$ is of similar form to that considered in Lemon and King [41] (with additional terms reflecting the viscosity of the water phase and dependence on nutrient concentration). The below calculation therefore follows that presented therein and so we omit much of the detail. We seek solutions of the form

$$
\theta_{n, 1}=N e^{\lambda t+i \gamma x}, v_{n, 1}=V e^{\lambda t+i \gamma x}, c_{T, 1}=C e^{\lambda t+i \gamma x},
$$

where $N, V, C$ are constants, $\gamma$ is the wavenumber and $\lambda$ the growth rate of the perturbation. We find that the growth rate is given by

$$
\lambda=\frac{1}{2}\left[G(\gamma)-H(\gamma) \pm \sqrt{(H(\gamma)-G(\gamma))^{2}-4(K-H(\gamma) G(\gamma))}\right]
$$


where

$$
\begin{aligned}
& E(\gamma)=\frac{\beta}{1-\theta_{n}^{*}}+\frac{4}{3} \gamma^{2}\left(1+\mu \frac{\theta_{n}^{*}}{1-\theta_{n}^{*}}\right) \\
& G(\gamma)=\frac{\partial S_{n}}{\partial \theta_{n}}\left(\theta_{n}^{*}, c^{*}\right)-\theta_{n}^{*} \gamma^{2} J\left(\theta_{n}^{*}\right) \frac{1}{E(\gamma)} \\
& H(\gamma)=\frac{1}{P e_{T}} \gamma^{2}+\frac{\partial \Lambda}{\partial c}\left(\theta_{n}^{*}, c^{*}\right) \\
& K=\frac{\partial \Lambda}{\partial \theta_{n}}\left(\theta_{n}^{*}, c^{*}\right) \frac{\partial S_{n}}{\partial c}\left(\theta_{n}^{*}, c^{*}\right) .
\end{aligned}
$$

Determination of the stability of the steady state requires values of the various model parameters as well as functions for growth $S_{n}$ and nutrient uptake $\Lambda$. We return to the stability in section 4 , where we seek illustrative numerical solutions, for given parameters and functions, as the stability of the non-trivial steady state plays an important role in the determination of the tissue growth behaviour.

\section{Multiple scales analysis}

We now work with the linearised model (2.27-2.31) and, for the sake of clarity, drop the associated subscripts. To derive a suitable macroscale description incorporating the microscale growth, dynamics and structure, we follow $[13,19,54,61,69]$ in using the method of multiple scales. Correspondingly we rescale equations $(2.27-2.31)$ such that the timescale under consideration is that of macroscale advection and pressure scaling results in the appropriate leading order problem:

$$
t=\varepsilon \tilde{t}, \quad p=\frac{1}{\varepsilon} \tilde{p}
$$

in which tildes denote the rescaled variables. We drop the tilde notation for convenience as we work exclusively with the rescaled variables in subsequent sections. This choice of time rescaling simplifies the analysis by resulting in a quasi-steady problem at leading order.

Next we introduce a macroscale coordinate $\boldsymbol{X}$ where $\boldsymbol{X}=\varepsilon \boldsymbol{x}$ ( $\boldsymbol{x}$ being the microscale coordinate). Since the two length scales are well-separated we treat $\boldsymbol{x}$ and $\boldsymbol{X}$ as independent and expand variables and differential operators in multiple-scales form as follows:

$$
\begin{aligned}
& \psi(\boldsymbol{x}, \boldsymbol{X}, t ; \varepsilon)=\psi^{(0)}(\boldsymbol{x}, \boldsymbol{X}, t)+\varepsilon \psi^{(1)}(\boldsymbol{x}, \boldsymbol{X}, t)+\ldots \\
& \boldsymbol{\nabla}=\boldsymbol{\nabla}_{\boldsymbol{x}}+\varepsilon \boldsymbol{\nabla}_{\boldsymbol{X}}, \quad \nabla^{2}=\nabla_{\boldsymbol{x}}^{2}+2 \varepsilon \boldsymbol{\nabla}_{\boldsymbol{x}} \cdot \boldsymbol{\nabla}_{\boldsymbol{X}}+\varepsilon^{2} \nabla_{\boldsymbol{X}}^{2} .
\end{aligned}
$$

Moreover, in addition to the boundary conditions (2.30-2.31) we assume that $\psi^{(i)}$ for $i=0,1, \ldots$ are periodic in $\boldsymbol{x}$. We now analyse the equations at each order in $\varepsilon$, with the aim of obtaining a description of the macroscale growth and transport. 


\section{$3.1 \quad \mathcal{O}(1)$}

The equations and boundary conditions at leading order are given below. In the tissue domain, $\Omega_{T}$ :

$$
\begin{aligned}
\theta_{n}^{*} \boldsymbol{\nabla}_{\boldsymbol{x}} \cdot \boldsymbol{v}_{n}^{(0)} & =S_{n}^{(0)}, \\
\theta_{w}^{*} \boldsymbol{\nabla}_{\boldsymbol{x}} \cdot \boldsymbol{v}_{w}^{(0)} & =-\bar{\rho} S_{n}^{(0)}, \\
\theta_{n}^{(0)}+\theta_{w}^{(0)} & =0, \\
\boldsymbol{\nabla}_{\boldsymbol{x}} \cdot \boldsymbol{v}_{T}^{(0)} & =(1-\bar{\rho}) S_{n}^{(0)}, \\
\theta_{n}^{*} \boldsymbol{\nabla}_{\boldsymbol{x}}\left(p_{w}^{(0)}+\phi_{n}^{(0)}\right) & =\mathbf{0}, \\
\theta_{w}^{*} \boldsymbol{\nabla}_{\boldsymbol{x}} p_{w}^{(0)} & =\mathbf{0}, \\
\boldsymbol{\nabla}_{\boldsymbol{x}} \cdot\left(c^{*} \boldsymbol{v}_{T}^{(0)}\right) & =\frac{1}{P e_{T}} \nabla_{\boldsymbol{x}}^{2} c_{T}^{(0)}-\Lambda^{(0)} .
\end{aligned}
$$

In the interstitial domain, $\Omega_{I}$ :

$$
\begin{aligned}
\boldsymbol{\nabla}_{\boldsymbol{x}} \cdot \boldsymbol{v}_{I}^{(0)} & =0, \\
\boldsymbol{\nabla}_{\boldsymbol{x}} p_{I}^{(0)} & =\mathbf{0}, \\
\boldsymbol{\nabla}_{\boldsymbol{x}} \cdot\left(c^{*} \boldsymbol{v}_{I}^{(0)}\right) & =\frac{1}{P e_{I}} \nabla_{\boldsymbol{x}}^{2} c_{I}^{(0)} .
\end{aligned}
$$

Boundary conditions on $\Gamma$ :

$$
\begin{gathered}
\theta_{n}^{*}\left(\boldsymbol{v}_{n}^{(0)}-\boldsymbol{v}_{\Gamma}^{(0)}\right) \cdot \boldsymbol{n}=0, \\
\theta_{w}^{*}\left(\boldsymbol{v}_{w}^{(0)}-\boldsymbol{v}_{\Gamma}^{(0)}\right) \cdot \boldsymbol{n}=\left(\boldsymbol{v}_{I}^{(0)}-\boldsymbol{v}_{\Gamma}^{(0)}\right) \cdot \boldsymbol{n}, \\
\boldsymbol{v}_{T}^{(0)} \cdot \boldsymbol{t}=\boldsymbol{v}_{I}^{(0)} \cdot \boldsymbol{t}, \\
-\left(p_{w}^{(0)}+\theta_{n}^{*} \phi_{n}^{(0)}\right) \boldsymbol{I}=-p_{I}^{(0)} \boldsymbol{I}, \\
\boldsymbol{\nabla}_{\boldsymbol{x}} F^{*} \cdot \boldsymbol{v}_{\Gamma}^{(0)}=0, \\
c^{*}\left(\boldsymbol{v}_{T}^{(0)}-\boldsymbol{v}_{\Gamma}^{(0)}\right) \cdot \boldsymbol{n}-\frac{1}{P e_{T}} \nabla_{\boldsymbol{x}} c_{T}^{(0)} \cdot \boldsymbol{n} \\
=c^{*}\left(\boldsymbol{v}_{I}^{(0)}-\boldsymbol{v}_{\Gamma}^{(0)}\right) \cdot \boldsymbol{n}-\frac{1}{P e_{I}} \nabla_{\boldsymbol{x}} c_{I}^{(0)} \cdot \boldsymbol{n}, \\
c_{T}^{(0)}=c_{I}^{(0)},
\end{gathered}
$$

where

$$
\boldsymbol{v}_{T}^{(0)}=\theta_{n}^{*} \boldsymbol{v}_{n}^{(0)}+\theta_{w}^{*} \boldsymbol{v}_{w}^{(0)}
$$


Boundary conditions on $\partial \Omega_{S}$ :

$$
\boldsymbol{v}_{n}^{(0)}=\boldsymbol{v}_{w}^{(0)}=\mathbf{0}, \quad \nabla_{\boldsymbol{x}} c_{T}^{(0)} \cdot \boldsymbol{n}_{S}=0 .
$$

Lastly, we note that, in view of (2.28), the phase transfer, intraphase pressure and nutrient uptake functions depend only on $\theta_{n}^{(0)}, c_{T}^{(0)}$ (and the relevant steady states), so that

$$
S_{n}^{(0)}=S_{n, 1}\left(\theta_{n}^{(0)}, c_{T}^{(0)}\right), \quad \phi_{n}^{(0)}=\phi_{n, 1}\left(\theta_{n}^{(0)}\right), \quad \Lambda^{(0)}=\Lambda_{1}\left(\theta_{n}^{(0)}, c_{T}^{(0)}\right) .
$$

Equation (3.6e) provides $\boldsymbol{\nabla}_{\boldsymbol{x}} F^{*}=\mathbf{0}$ or $\boldsymbol{v}_{\Gamma}^{(0)} \cdot \boldsymbol{n}=0$, but the latter holds most generally and so the tissue-interstitium boundary is stationary at leading order. Correspondingly, we rescale mass-transfer and nutrient consumption to $O(\varepsilon)$ (as in other multiscale models [19, 54]). Note that this occurs as a consequence of the scaling choice (3.1).

Equations $(3.4 \mathrm{e}, \mathrm{f})$ and $(3.5 \mathrm{~b})$ imply that the leading order pressures are independent of the microscale coordinate $\boldsymbol{x}$. Using the boundary condition (3.6d) we find that the pressure everywhere is given by

$$
p^{(0)}(\boldsymbol{X}, t)=p_{I}^{(0)}(\boldsymbol{X}, t)=p_{w}^{(0)}(\boldsymbol{X}, t)+\theta_{n}^{*} \phi_{n}^{(0)}(\boldsymbol{X}, t) .
$$

Moreover, in view of the above rescaling, and following the argument in [54],

$$
c^{(0)}(\boldsymbol{X}, t)=c_{T}^{(0)}(\boldsymbol{X}, t)=c_{I}^{(0)}(\boldsymbol{X}, t)
$$

and, furthermore, $\phi_{n}^{(0)}=\phi_{n}^{(0)}(\boldsymbol{X}, t), \theta_{n}^{(0)}=\theta_{n}^{(0)}(\boldsymbol{X}, t)$.

\section{$3.2 \mathcal{O}(\varepsilon)$}

The equations and boundary conditions at $\mathcal{O}(\varepsilon)$ are given below. In $\Omega_{T}$ :

$$
\begin{gathered}
\frac{\partial \theta_{n}^{(0)}}{\partial t}+\theta_{n}^{*}\left(\boldsymbol{\nabla}_{\boldsymbol{x}} \cdot \boldsymbol{v}_{n}^{(1)}+\boldsymbol{\nabla}_{\boldsymbol{X}} \cdot \boldsymbol{v}_{n}^{(0)}\right)=S_{n}^{(0)}, \\
\boldsymbol{\nabla}_{\boldsymbol{x}} \cdot \boldsymbol{v}_{T}^{(1)}+\boldsymbol{\nabla}_{\boldsymbol{X}} \cdot \boldsymbol{v}_{T}^{(0)}=(1-\bar{\rho}) S_{n}^{(0)}, \\
\theta_{n}^{*}\left[\boldsymbol{\nabla}_{\boldsymbol{x}}\left(p_{w}^{(1)}+\phi_{n}^{(1)}\right)+\boldsymbol{\nabla}_{\boldsymbol{X}}\left(p_{w}^{(0)}+\phi_{n}^{(0)}\right)+\beta \theta_{w}^{*}\left(\boldsymbol{v}_{n}^{(0)}-\boldsymbol{v}_{w}^{(0)}\right)-\nabla_{\boldsymbol{x}}^{2} \boldsymbol{v}_{n}^{(0)}\right]=\mathbf{0}, \\
\theta_{w}^{*}\left[\boldsymbol{\nabla}_{\boldsymbol{x}} p_{w}^{(1)}+\boldsymbol{\nabla}_{\boldsymbol{X}} p_{w}^{(0)}+\beta \theta_{n}^{*}\left(\boldsymbol{v}_{w}^{(0)}-\boldsymbol{v}_{n}^{(0)}\right)-\mu \nabla_{\boldsymbol{x}}^{2} \boldsymbol{v}_{w}^{(0)}\right]=\mathbf{0}, \\
\frac{\partial c^{(0)}}{\partial t}+c^{*}\left(\boldsymbol{\nabla}_{\boldsymbol{x}} \cdot \boldsymbol{v}_{T}^{(1)}+\boldsymbol{\nabla}_{\boldsymbol{X}} \cdot \boldsymbol{v}_{T}^{(0)}\right)=\frac{1}{P e_{T}} \nabla_{\boldsymbol{x}}^{2} c_{T}^{(1)}-\Lambda^{(0)},
\end{gathered}
$$

where $\boldsymbol{v}_{T}^{(1)}=\theta_{n}^{*} \boldsymbol{v}_{n}^{(1)}+\theta_{w}^{*} \boldsymbol{v}_{w}^{(1)}$. In $\Omega_{I}$ :

$$
\begin{aligned}
\boldsymbol{\nabla}_{\boldsymbol{x}} \cdot \boldsymbol{v}_{I}^{(1)}+\boldsymbol{\nabla}_{\boldsymbol{X}} \cdot \boldsymbol{v}_{I}^{(0)} & =0 \\
\boldsymbol{\nabla}_{\boldsymbol{x}} p_{I}^{(1)}+\boldsymbol{\nabla}_{\boldsymbol{X}} p^{(0)}-\mu \nabla_{\boldsymbol{x}}^{2} \boldsymbol{v}_{I}^{(0)} & =\mathbf{0}, \\
\frac{\partial c^{(0)}}{\partial t}+c^{*}\left(\boldsymbol{\nabla}_{\boldsymbol{x}} \cdot \boldsymbol{v}_{I}^{(1)}+\boldsymbol{\nabla}_{\boldsymbol{X}} \cdot \boldsymbol{v}_{I}^{(0)}\right) & =\frac{1}{P e_{I}} \nabla_{\boldsymbol{x}}^{2} c_{I}^{(1)} .
\end{aligned}
$$


On $\Gamma$ :

$$
\begin{gathered}
\theta_{n}^{*}\left(\boldsymbol{v}_{n}^{(1)}-\boldsymbol{v}_{\Gamma}^{(1)}\right) \cdot \boldsymbol{n}=0 \\
\theta_{w}^{*}\left(\boldsymbol{v}_{w}^{(1)}-\boldsymbol{v}_{\Gamma}^{(1)}\right) \cdot \boldsymbol{n}=\left(\boldsymbol{v}_{I}^{(1)}-\boldsymbol{v}_{\Gamma}^{(1)}\right) \cdot \boldsymbol{n}, \\
\boldsymbol{v}_{T}^{(1)} \cdot \boldsymbol{t}=\boldsymbol{v}_{I}^{(1)} \cdot \boldsymbol{t}, \\
-\left(p_{w}^{(1)}+\theta_{n}^{*} \phi_{n}^{(1)}\right) \boldsymbol{I}+\theta_{n}^{*}\left(\boldsymbol{\nabla}_{\boldsymbol{x}} \boldsymbol{v}_{n}^{(0)}+\left(\boldsymbol{\nabla}_{\boldsymbol{x}} \boldsymbol{v}_{n}^{(0)}\right)^{\top}\right) \\
+\mu \theta_{w}^{*}\left(\boldsymbol{\nabla}_{\boldsymbol{x}} \boldsymbol{v}_{w}^{(0)}+\left(\boldsymbol{\nabla}_{\boldsymbol{x}} \boldsymbol{v}_{w}^{(0)}\right)^{\top}\right)=-p_{I}^{(1)} \boldsymbol{I}+\mu\left(\boldsymbol{\nabla}_{\boldsymbol{x}} \boldsymbol{v}_{I}^{(0)}+\left(\boldsymbol{\nabla} \boldsymbol{v}_{I}^{(0)}\right)^{T}\right) \\
\frac{\partial F^{(0)}}{\partial t}+\boldsymbol{\nabla}_{\boldsymbol{x}} F^{*} \cdot \boldsymbol{v}_{\Gamma}^{(1)}=0 \\
c^{*}\left(\boldsymbol{v}_{T}^{(1)}-\boldsymbol{v}_{\Gamma}^{(1)}\right) \cdot \boldsymbol{n}-\frac{1}{P e_{T}}\left(\boldsymbol{\nabla}_{\boldsymbol{x}} c_{T}^{(1)}+\boldsymbol{\nabla}_{\boldsymbol{X}} c^{(0)}\right) \cdot \boldsymbol{n} \\
=c^{*}\left(\boldsymbol{v}_{I}^{(1)}-\boldsymbol{v}_{\Gamma}^{(1)}\right) \cdot \boldsymbol{n}-\frac{1}{P e_{I}}\left(\boldsymbol{\nabla}_{\boldsymbol{x}} c_{I}^{(1)}+\boldsymbol{\nabla}_{\boldsymbol{X}} c^{(0)}\right) \cdot \boldsymbol{n},
\end{gathered}
$$

On $\partial \Omega_{S}$ :

$$
\boldsymbol{v}_{n}^{(1)}=\boldsymbol{v}_{w}^{(1)}=\mathbf{0}, \quad \nabla_{\boldsymbol{x}} c_{T}^{(1)} \cdot \boldsymbol{n}+\nabla_{\boldsymbol{X}} c_{T}^{(0)} \cdot \boldsymbol{n}_{S}=0
$$

\subsection{Macroscale equations}

\subsubsection{Ansatz}

In what follows we require a method of averaging variables across the various domains of the periodic cell. We therefore define the following integral average for some variable, $g$, over domain $\Omega_{i}$, by

$$
\langle g\rangle_{i}=\frac{1}{|\Omega|} \int_{\Omega_{i}} g \mathrm{~d} V,
$$

where $\Omega=\Omega_{I} \cup \Omega_{T} \cup \Omega_{S}$. Due to the linearity of the momentum equations, we take an appropriate form for the macroscale velocities and microscale pressures to be given by

$$
\boldsymbol{v}_{i}^{(0)}=-\boldsymbol{K}_{i} \boldsymbol{\nabla}_{\boldsymbol{X}} p^{(0)} \text { and } p_{i}^{(1)}=-\boldsymbol{a}_{i} \boldsymbol{\nabla}_{\boldsymbol{X}} p^{(0)}-\bar{p}_{i} \text { for } i=n, w, I,
$$


where $\boldsymbol{K}_{i}$ are tensors describing the permeability, $\bar{p}_{i}$ are the mean values of the first order pressures in $\Omega_{i}$, hence microscale invariant, and $\boldsymbol{a}_{i}$ are first order tensors imparting microscale variation; see, for example, [22, 54, 69].

Substituting (3.17) into the mass and momentum conservation equations (3.4a, 3.4b, 3.5a, 3.12c, 3.12d, 3.13b) and boundary conditions (3.6a-c, 3.14d), provides the following 'cell problems' that determine $\boldsymbol{K}_{i}$ and $\boldsymbol{a}_{i}$ in each domain. A 'cell problem' is one posed on the microscale periodic unit or 'cell' that makes up the macroscale domain, from which one establishes the microscale variation of certain quantities (here, the permeability) in order to specify effective coefficients in the macroscale description. For example, here Figure 1 illustrates sample periodic cells. In $\Omega_{I}$ :

$$
\begin{gathered}
\nabla_{\boldsymbol{x}} \cdot \boldsymbol{K}_{I}^{\top}=\mathbf{0}, \\
\nabla_{\boldsymbol{x}} \boldsymbol{a}_{I}^{\top}-\boldsymbol{I}-\mu \nabla_{\boldsymbol{x}}^{2} \boldsymbol{K}_{I}=\mathbf{0} .
\end{gathered}
$$

In $\Omega_{T}$ :

$$
\begin{gathered}
\boldsymbol{\nabla}_{\boldsymbol{x}} \cdot \boldsymbol{K}_{n}^{\top}=\mathbf{0}, \quad \boldsymbol{\nabla}_{\boldsymbol{x}} \cdot \boldsymbol{K}_{w}^{\top}=\mathbf{0} \\
{\left[\boldsymbol{\nabla}_{\boldsymbol{x}} \boldsymbol{a}_{w}^{\top}-\boldsymbol{I}-\mu \nabla_{\boldsymbol{x}}^{2} \boldsymbol{K}_{w}-\beta \theta_{n}^{*}\left(\boldsymbol{K}_{n}-\boldsymbol{K}_{w}\right)\right] \boldsymbol{\nabla}_{\boldsymbol{X}} p^{(0)}+\theta_{n}^{*} \boldsymbol{\nabla}_{\boldsymbol{X}} \phi_{n}^{(0)}=\mathbf{0}} \\
{\left[\boldsymbol{\nabla}_{\boldsymbol{x}} \boldsymbol{a}_{n}^{\top}-\boldsymbol{I}-\nabla_{\boldsymbol{x}}^{2} \boldsymbol{K}_{n}-\beta \theta_{w}^{*}\left(\boldsymbol{K}_{w}-\boldsymbol{K}_{n}\right)\right] \boldsymbol{\nabla}_{\boldsymbol{X}} p^{(0)}-\theta_{w}^{*} \boldsymbol{\nabla}_{\boldsymbol{X}} \phi_{n}^{(0)}=\mathbf{0} .}
\end{gathered}
$$

On $\Gamma$ :

$$
\begin{gathered}
\boldsymbol{K}_{i}^{\top} \boldsymbol{n}=\mathbf{0} \quad \text { for } i=n, w, I \\
\left(\theta_{n}^{*} \boldsymbol{K}_{n}^{\top}+\theta_{w}^{*} \boldsymbol{K}_{w}^{\top}\right) \boldsymbol{t}=\boldsymbol{K}_{I}^{\top} \boldsymbol{t} \\
-\boldsymbol{a}_{T} \otimes \boldsymbol{I}+\left(\boldsymbol{\nabla} \boldsymbol{K}_{T}+\left(\nabla \boldsymbol{K}_{T}\right)^{\top}\right)=-\boldsymbol{a}_{I} \otimes \boldsymbol{I}+\mu\left(\boldsymbol{\nabla} \boldsymbol{K}_{I}+\left(\nabla \boldsymbol{K}_{I}\right)^{\top}\right)
\end{gathered}
$$

where the microscale invariant contributions to stress are continuous, i.e. $\bar{p}_{I}=\theta_{n}^{*} \bar{p}_{n}+\theta_{w}^{*} \bar{p}_{w}$ and

$$
\boldsymbol{K}_{T}=\theta_{n}^{*} \boldsymbol{K}_{n}+\mu \theta_{w}^{*} \boldsymbol{K}_{w} \quad \text { and } \quad \boldsymbol{a}_{T}=\theta_{n}^{*} \boldsymbol{a}_{n}+\theta_{w}^{*} \boldsymbol{a}_{w} .
$$

Finally, on $\partial \Omega_{S}$ :

$$
\boldsymbol{K}_{n}=\mathbf{0}, \quad \boldsymbol{K}_{w}=\mathbf{0} .
$$

For uniqueness in the 'cell problems', we use a standard approach [50, 54, $61,69]$ and impose that in the relevant domain

$$
\left\langle\boldsymbol{a}_{i}\right\rangle=\mathbf{0},
$$


i.e. that the average of the microscale variation in pressure, imparted by $\boldsymbol{a}_{i}$, is zero over the volume of the periodic cell.

We note that, while a standard Stokes-type cell problem is obtained in $\Omega_{I}$, the multiphase dynamics in $\Omega_{T}$ leads to significantly increased complexity. In particular, we obtain a pair of coupled modified Stokes problems, determining the permeability tensors $\boldsymbol{K}_{i}$ and extra pressures $\boldsymbol{a}_{i}$ for each phase, which are further coupled to the flow in $\Omega_{I}$ via stress and velocity continuity boundary conditions. Moreover, and in contrast to other simplified analyses of this type, the microscale cell problems and corresponding macroscale dynamics are coupled through the macroscale pressure and active cell behaviour terms. Such a multiscale cell problem poses significant computational challenges; in section 5 we present illustrative numerical simulations in a simplified limit case. Methodologies for computations of the full multiscale problem are discussed in section 6 .

\subsubsection{Averaging}

Averaging equations (3.13a) and (3.12b), using the definition in (3.16), then applying the divergence theorem we find

$$
\begin{aligned}
& \nabla_{\boldsymbol{X}} \cdot\left\langle\boldsymbol{v}_{I}^{(0)}\right\rangle_{I}=\frac{1}{|\Omega|} \int_{\Gamma} \boldsymbol{v}_{I}^{(1)} \cdot \boldsymbol{n} \mathrm{d} S, \\
& \boldsymbol{\nabla}_{\boldsymbol{X}} \cdot\left\langle\boldsymbol{v}_{T}^{(0)}\right\rangle_{T}=-\frac{1}{|\Omega|} \int_{\Gamma} \boldsymbol{v}_{T}^{(1)} \cdot \boldsymbol{n} \mathrm{d} S+\left\langle(1-\bar{\rho}) S_{n}^{(0)}\right\rangle_{T} .
\end{aligned}
$$

Note that the contribution from the scaffold-tissue boundary arising in $(3.12 \mathrm{~b})$ is zero since all phase velocities are zero there. Using boundary conditions (3.14a) and (3.14b) and defining the average macroscale velocity by

$$
\overline{\boldsymbol{v}}^{(0)}=\left\langle\boldsymbol{v}_{T}^{(0)}\right\rangle_{T}+\left\langle\boldsymbol{v}_{I}^{(0)}\right\rangle_{I},
$$

we obtain

$$
\boldsymbol{\nabla}_{\boldsymbol{X}} \cdot \overline{\boldsymbol{v}}^{(0)}=\left\langle(1-\bar{\rho}) S_{n}^{(0)}\right\rangle_{T} .
$$

In view of (3.17), this yields an equation for the macroscale pressure,

$$
\boldsymbol{\nabla}_{\boldsymbol{X}} \cdot\left(\overline{\boldsymbol{K}} \nabla_{\boldsymbol{X}} p^{(0)}\right)=-\left\langle(1-\bar{\rho}) S_{n}^{(0)}\right\rangle_{T},
$$

where

$$
\overline{\boldsymbol{K}}=\left\langle\theta_{n}^{*} \boldsymbol{K}_{n}+\theta_{w}^{*} \boldsymbol{K}_{w}\right\rangle_{T}+\left\langle\boldsymbol{K}_{I}\right\rangle_{I} .
$$

Averaging the nutrient concentration equations (3.12e) and (3.13c) over their respective domains gives

$$
\begin{aligned}
& \frac{\left|\Omega_{T}\right|}{|\Omega|} \frac{\partial c^{(0)}}{\partial t}+c^{*}(1-\bar{\rho})\left\langle S_{n}^{(0)}\right\rangle_{T}=\frac{1}{P e_{T}}\left\langle\nabla_{\boldsymbol{x}}^{2} c_{T}^{(1)}\right\rangle_{T}-\left\langle\Lambda^{(0)}\right\rangle_{T}, \\
& \frac{\left|\Omega_{I}\right|}{|\Omega|} \frac{\partial c^{(0)}}{\partial t}=\frac{1}{P e_{I}}\left\langle\nabla_{\boldsymbol{x}}^{2} c_{I}^{(1)}\right\rangle_{I} .
\end{aligned}
$$


The sum of (3.35) and (3.36), after the application of the divergence theorem and boundary condition (3.14f), yields the macroscale nutrient concentration equation,

$$
\frac{\left|\Omega_{T} \cup \Omega_{I}\right|}{|\Omega|} \frac{\partial c^{(0)}}{\partial t}+c^{*}(1-\bar{\rho})\left\langle S_{n}^{(0)}\right\rangle_{T}=-\left\langle\Lambda^{(0)}\right\rangle_{T} .
$$

Finally, averaging the equation for the cell volume fraction (3.12a) and applying the divergence theorem and boundary condition (3.14a) we find

$$
\frac{\partial}{\partial t}\left\langle\theta_{n}^{(0)}\right\rangle_{T}+\theta_{n}^{*}\left(\boldsymbol{\nabla}_{\boldsymbol{X}} \cdot\left\langle\boldsymbol{v}_{n}^{(0)}\right\rangle_{T}+\left\langle\boldsymbol{v}_{\Gamma}^{(1)} \cdot \boldsymbol{n}\right\rangle_{\Gamma}\right)=\left\langle S_{n}^{(0)}\right\rangle_{T}
$$

\subsubsection{Summary}

From an underlying pore-scale multiphase tissue growth model, we have derived a macroscale description that incorporates explicitly the microscale structure and dynamics. The microscale problem comprises a set of coupled Stokes problems (3.18-3.27) that provide permeability tensors with which to parameterise the macroscale Darcy flow PDE (3.33) and thereby the reaction equations describing tissue component volume fractions (3.38) and nutrient concentration (3.37). In common with other studies of this type, tissue growth at the microscale influences macroscale dynamics by inducing a leading order flow and by source and sink terms in the equations governing the phase volume fraction and nutrient concentration. In contrast, however, we note that the permeability tensor $\overline{\boldsymbol{K}}$ retains multiscale dependence through the pressure and cell motility forcing that appears in $(3.20,3.21)$ and so, despite the simplified analysis that we employ, the micro- and macro-scale problems remain fully coupled. This increase in complexity is somewhat offset by the fact that the macroscale formulation simplifies significantly, with the resulting evolution equations for $\theta_{n}$ and $c$ containing no spatial component, except that imbued by initial data.

We remark that in many biological applications the density differences between phases may be small (and so terms in $(3.32,3.33,3.37$ ) associated with the corresponding change in volume as mass transfer occurs will vanish) and that these are often neglected in the tissue growth literature. As in Collis et al. [19], in the following, we choose to retain non-infinitesimal density differences in the interests of generality, and to highlight the full model dynamics. Lastly, we note that as is common in analyses of this type, the macroscale model we obtain is not closed: we are required to specify constitutively the $O(\varepsilon)$ boundary velocity $\boldsymbol{v}_{\Gamma}^{(1)} \cdot \boldsymbol{n}$ (cf. [19, 34]). This is explored in the following section; and in section 5 is illustrated in the special case of inviscid water, under which simplification, the Stokes problem loses its dependence on the macroscale pressures. 


\section{Closure of macroscale model}

In order to close the macroscale model we require information on the boundary velocity, $\boldsymbol{v}_{\Gamma}^{(1)} \cdot \boldsymbol{n}$. In principle this can be obtained by considering a suitable free-boundary problem. Instead, we choose to specify $\boldsymbol{v}_{\Gamma}^{(1)} \cdot \boldsymbol{n}$ constitutively. To provide a rationale we investigate numerically the full non-linear model (see section 2.1) in one spatial dimension, to explore the impact of various model parameters on tissue growth, thereby gaining insight in to the qualitative behaviour of growth in the multiphase context and informing the choice of a suitable constitutive equation for the boundary velocity.

The multiphase model of [42], on which our tissue description is based, was further investigated in [41], concentrating on its travelling wave behaviour. The variation of the wave speed with the model parameters was investigated, using both analytical and numerical methods. Due to the similarities between their model and our own, and the correspondence between such travelling wave behaviour and the free boundary motion $\boldsymbol{v}_{\Gamma}^{(1)} \cdot \boldsymbol{n}$, we follow a similar approach here. We provide brief summaries of various methods employed but for further detail refer the reader to [41]. Note that unlike [41] we do not include the scaffold as a component of the multiphase mixture. Also, we initially include viscosity of the water phase, before setting $\mu_{w}=0$ in the numerical experiments.

Since in the multiscale model, the concentration of nutrient is independent of the microscale at leading order, here we correspondingly assume that $c$ is constant across the tissue. Lastly, we remark that for simplicity, and consistency with [41], we consider the case for which $\rho_{n}=\rho_{w}$ and concentrate instead on the dependence of travelling wave speed on the cells' motile properties. Our simplified setup is illustrated in Figure 2.

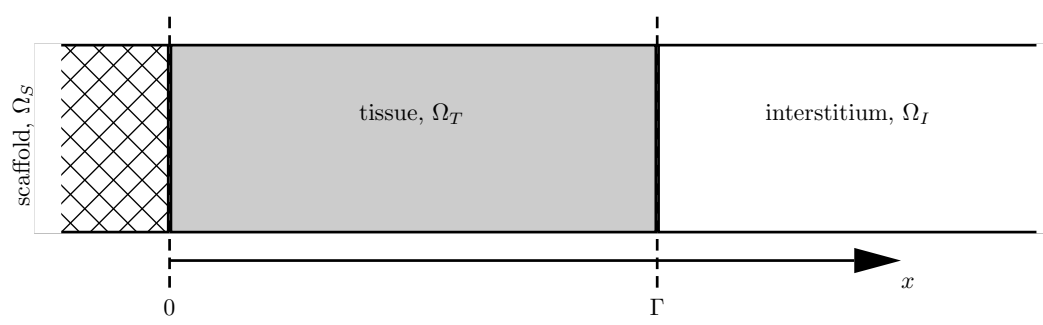

Figure 2: Illustration to show the three domains for the 1D model. The scaffold-tissue boundary at $x=0$ is fixed while the tissue-interstitial boundary at $x=\Gamma$ is a free interface whose motion arises due to growth and active cell motion. 


\subsection{Model equations and numerical method}

In $\Omega_{I}$ the equations $(2.13,2.14)$ in one dimension reduce $v_{I}$ and $p_{I}$ to constants which, assuming $v_{I}, p_{I} \rightarrow 0$ as $x \rightarrow \infty$, implies $v_{I}=p_{I}=0$.

In the tissue domain, elimination of $\theta_{w}, v_{w}$ and $p_{w}$ reduces the governing equations $(2.2-2.4,2.9,2.10)$ in $1 \mathrm{D}$ to

$$
\begin{gathered}
\frac{\partial \theta_{n}}{\partial t}+\frac{\partial}{\partial x}\left(\theta_{n} v_{n}\right)=S_{n}, \\
A \frac{\partial^{2} v_{n}}{\partial x^{2}}+B \frac{\partial v_{n}}{\partial x}+C v_{n}=D,
\end{gathered}
$$

where

$$
\begin{aligned}
A & =\frac{4}{3} \theta_{n}\left(\mu_{n}+\left(\mu_{w}-\mu_{n}\right) \theta_{n}\right), \\
B & =\frac{4}{3} \frac{\partial \theta_{n}}{\partial x} \frac{1}{1-\theta_{n}}\left(\left(\mu_{n}-\mu_{w}\right) \theta_{n}^{2}+2\left(\mu_{w}-\mu_{n}\right) \theta_{n}+\mu_{n}\right), \\
C & =-\beta \theta_{n}+\frac{4}{3} \frac{\mu_{w} \theta_{n}}{1-\theta_{n}}\left(\frac{\partial^{2} \theta_{n}}{\partial x^{2}}+\frac{1}{1-\theta_{n}}\left(\frac{\partial \theta_{n}}{\partial x}\right)^{2}\right), \\
D & =\left(1-\theta_{n}\right)\left(-2 \nu \theta_{n}+\frac{\kappa \theta_{n}^{2}\left(3-2 \theta_{n}\right)}{\left(1-\theta_{n}\right)^{2}}\right) \frac{\partial \theta_{n}}{\partial x},
\end{aligned}
$$

and following [41] we choose

$$
S_{n}=k_{m} c \theta_{n}\left(1-\theta_{n}\right)-k_{n} \theta_{n} .
$$

Suitable boundary conditions (see [41]) are given by

$$
\begin{array}{lll}
\text { at } x=0: & \frac{\partial \theta_{n}}{\partial x}=0, & v_{n}=0, \\
\text { at } x=\Gamma: & \theta_{n}=0, & \frac{\partial v_{n}}{\partial x}=0 .
\end{array}
$$

For consistency with the multiscale numerical experiments that follow, we investigate the behaviour when the water phase is inviscid $\left(\mu_{w}=0\right)$. Such a simplification is common in multiphase studies of tissue dynamics [1] and is consistent with [41], in particular.

To solve (4.1) we use a semidiscrete MUSCL (Monotonic Upstreamcentered Scheme for Conservation Laws) scheme [74], a finite volume scheme, with monotonized central (MC) slope limiter [74] and upwind flux. At each time step the finite difference form of (4.2) is used to calculate the cell velocity given the current solution for $\theta_{n}$. The resulting system is timestepped via ode23.m, a Runge-Kutta method with adaptive step size, in MATLAB. To aid the solution of (4.2), we adopt an approach from the study of dry-bed dam-break problems, described in [32], as follows. A 
small tolerance value for the cell volume fraction, $\theta_{t o l}$, is chosen such that if $\theta_{n}<\theta_{t o l}$ on both sides of a finite volume interface, no flux is permitted. The cutoff value is chosen such that the behaviour of $\theta_{n}$ and $v_{n}$ is not significantly impacted. Throughout this section we use $\theta_{t o l}=0.001$.

In the following numerics we use the parameter values given in Table 1 unless otherwise indicated. These values are based on those in [41] where one can find a discussion on how these are obtained. The initial condition, a flat region at the steady state value for cell volume fraction with a smooth yet relatively steep drop to zero at the edge, is given by

$$
\begin{gathered}
\theta_{n}(x, 0)=\theta^{*}\left[\left(1-\left[\frac{x-0.45}{0.05}\right]\right)^{2}[\mathcal{H}(x-0.45)-\mathcal{H}(x-0.5)]\right. \\
+1-\mathcal{H}(x-0.45)]
\end{gathered}
$$

where $\mathcal{H}$ is the Heaviside step function.

Table 1: Parameter values employed in the microscale numerical experiment unless stated otherwise, for equations (4.1-4.7). Values for $k_{m}$ and $k_{n}$ taken from [41], determined by fitting a similar model to experimental data of chondrocytes seeded on porous scaffolds. Other values are chosen based on the range explored in [41], with alternative values for each parameter investigated in the following analysis.

\begin{tabular}{cc}
\hline Parameter & Value \\
\hline$k_{m}$ & $1.8 \times 10^{-4}$ \\
$k_{n}$ & $3.4 \times 10^{-5}$ \\
$\nu$ & $1.2 \times 10^{-2}$ \\
$\kappa$ & $8.0 \times 10^{-3}$ \\
$\beta$ & $1.0 \times 10^{4}$ \\
$c$ & 1 \\
$\mu_{n}$ & 1 \\
\hline
\end{tabular}

\subsection{Travelling wave speed}

Numerical simulations (Figure 3) display three different behaviours of the tissue: forward or backward travelling waves and break up of the tissue, independent of the choice of initial condition, as observed in the similar model of Lemon and King [41]. The break up of tissue, illustrated by Figures 3(e,f), occurs when the choice of parameters leads to the non-trivial steady state becoming unstable, i.e. the parameter values give a positive perturbation growth rate (2.38). 
As may be observed from the numerical results, the wave-front takes the form of a fast-varying transition to zero. The boundary layer analysis of [41] provides an equation defining the cell volume fraction at the inner edge of the moving layer, termed the aggregate density, which satisfies:

$$
\nu \theta_{n}^{\dagger 2}-\frac{\kappa \theta_{n}^{\dagger 3}}{1-\theta_{n}^{\dagger}}+\frac{4}{3} \theta_{n}^{\dagger}\left(k_{m}\left(1-\theta_{n}^{\dagger}\right)-k_{n}\right)=0 .
$$

For fixed mitotic and necrotic rates, $k_{m}$ and $k_{n}$, the value of the aggregate density $\theta_{n}^{\dagger}$ depends on the ratio of the cells aggregation and repulsion strength. The wave direction is determined by the relationship between the non-trivial steady state, $\theta_{n}^{*}=1-k_{n} / k_{m}$ and $\theta_{n}^{\dagger}$. For $\theta_{n}^{\dagger}<\theta_{n}^{*}$ forward waves are observed (Figures $3(\mathrm{a}, \mathrm{b})$ ) whilst increasing $\nu$ relative to $\kappa$ leads to $\theta_{n}^{\dagger}>\theta_{n}^{*}$ and the direction reverses (Figures $3(\mathrm{c}, \mathrm{d})$ ).

These regions, of forward, backward and unstable waves, across $(\nu, \kappa)$ parameter space are illustrated by Figure 4(a). The choice of initial condition only determines the initial transient behaviour of the wave; subsequent behaviour depends on the parameter values relative to each other.

Since our focus is on tissue growth in the numerical experiments that follow we confine our choice of parameters to those which result in forward travelling waves. Moreover we remark that the numerical solutions become badly behaved in the other two cases, due to the steepening of the leading edge. Note that the results for the backward and unstable cases, Figures $3(\mathrm{c}-\mathrm{f})$, correspond to shorter time spans than for the forward travelling wave, because of these numerical difficulties.

Following the analysis of [41], we find that approximate expressions for the wave speed can be obtained in two particular cases. Firstly, when the cell volume fraction does not vary appreciably through the wavefront, when $\theta_{n}^{*} \approx \theta_{n}^{\dagger}$, one can linearise the equations about

$$
\theta_{n}=\theta_{n}^{*}, v_{n}=\frac{\partial v_{n}}{\partial x}=0 .
$$

Rearranging the linearised equations and solving provides the following boundary velocity approximation:

$$
v_{\Gamma} \approx \frac{S_{n}\left(\theta_{n}^{\dagger}\right)}{\theta_{n}^{\dagger}} \sqrt{\frac{4-3 \theta_{n}^{*} J\left(\theta_{n}^{*}\right) / S_{n}^{\prime}\left(\theta_{n}^{*}\right)}{3 \beta\left(1-\theta_{n}^{*}\right)^{-1}}} .
$$

Secondly, an approximation can be obtained in the case where there is no interphase drag. Here, numerical solutions show that the wave profiles attain a constant cell volume fraction of $\theta_{n}^{\dagger}$ and constant velocity gradient $\partial v_{n} / \partial x$. Substituting this information into (4.1) and integrating shows that

$$
v_{\Gamma} \approx \frac{S_{n}\left(\theta_{n}^{\dagger}\right)}{\theta_{n}^{\dagger}} \Gamma(t),
$$




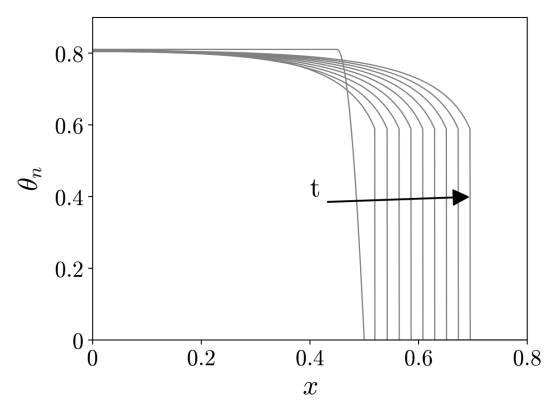

(a)

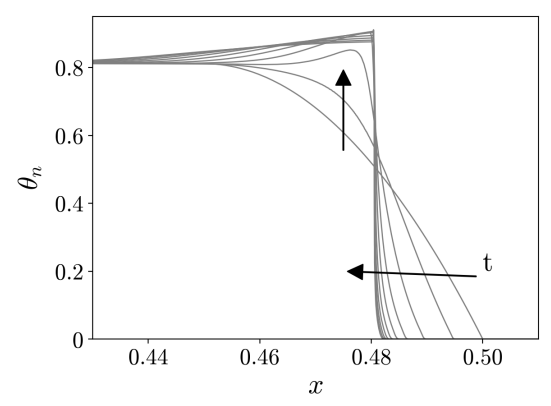

(c)

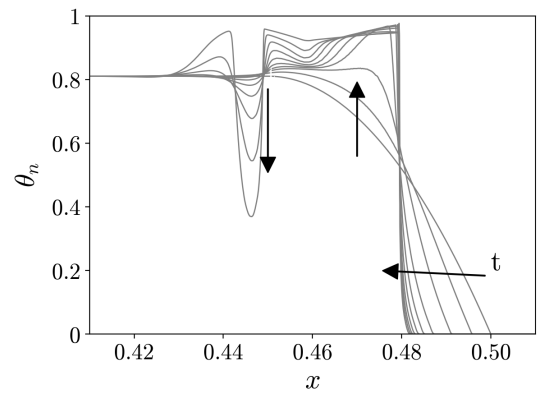

(e)

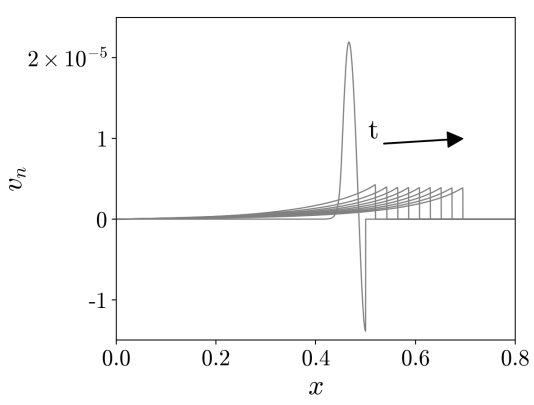

(b)

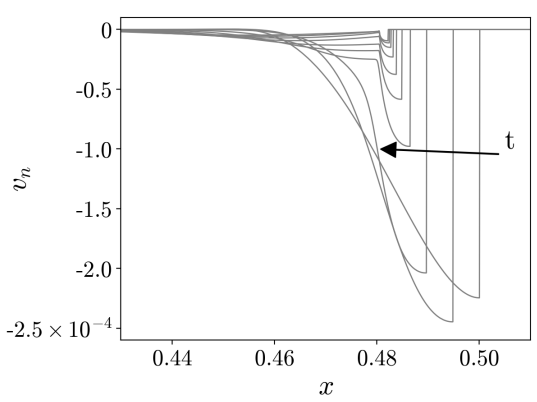

(d)

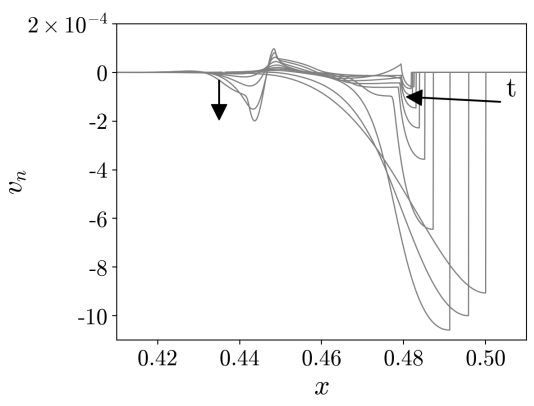

(f)

Figure 3: Cell volume fraction distribution and cell velocity obtained from numerical simulations of equations (4.1-4.7) for increasing time, showing the changing behaviour of the tissue as, for fixed repulsion strength, the aggregation parameter is increased. For low values, forward-travelling waves occur, while for increased aggregation the waves travel backwards and even higher values cause the break up of the tissue, due to the instability of the non-trivial steady state. Parameter values: (a), (b) $\nu=0.012, t=50000$, (c), (d) $\nu=0.08, t=200$, (e), (f) $\nu=0.3, t=40$. All other parameters as given in Table 1. 


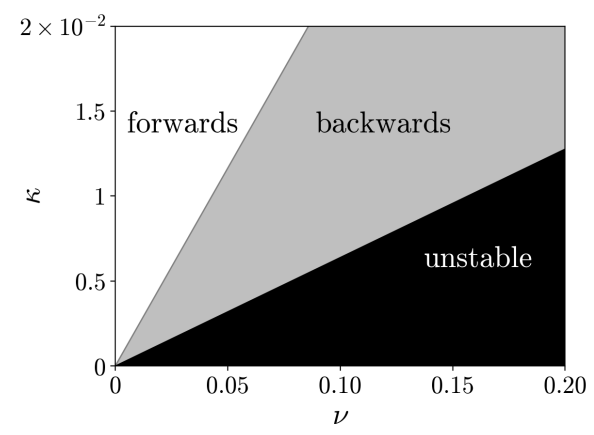

(a)

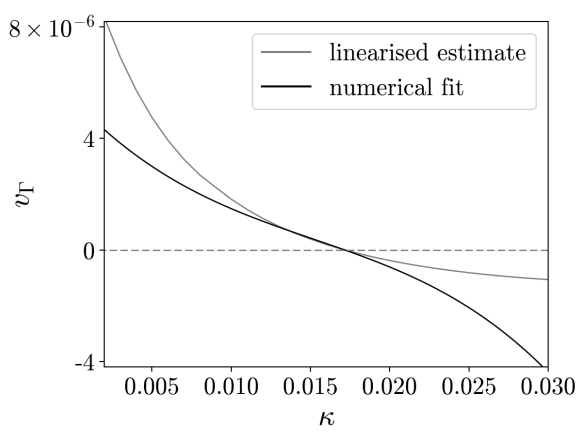

(b)

Figure 4: (a) Regions of $(\nu, \kappa)$ space where forward travelling, backwards travelling and unstable waves occur. Lines for stationary waves (separating white and grey region) and stability (separating grey and black region) are plotted using (4.10) (with $\theta^{\dagger}=\theta^{*}$ ) and (2.38). (b) Comparison of (4.13) (black) with linear estimate (4.11) (grey) for boundary velocity. Parameter values: $\kappa=4 \times 10^{-3}$. All other parameters for both figures as given in Table 1.

and hence the tissue grows, and the boundary velocity increases, exponentially.

Henceforth we employ the above results to help rationalise a more general constitutive representation for the boundary velocity $\boldsymbol{v}_{\Gamma}^{(1)} \cdot \boldsymbol{n}$ of the growing tissue domain $\Omega_{T}$ in the multiscale model presented in section 3.3.2. We reiterate that since our focus is tissue growth, we focus on forward travelling waves. We computed the travelling wave velocity for a range of parameter values (concentrating on $\nu, \kappa, \beta$ and nutrient concentration $c$, with the remainder chosen to be consistent with [41], as given in Table 1). Then, by employing MATLAB's curve fitting tool, a suitable estimate for the boundary velocity for this one dimensional model is given by

$$
v_{\Gamma}=\frac{100}{\sqrt{\beta}}\left(\frac{c-c^{*}}{1-c^{*}}\right)\left[\gamma_{1} \kappa^{\alpha_{1}} \nu^{3}+\gamma_{2} \kappa^{\alpha_{2}} \nu^{2}+\gamma_{3} \kappa^{\alpha_{3}} \nu+\gamma_{3} \kappa^{\alpha_{4}}\right]
$$

where

$$
c^{*}=\frac{k_{n}(\nu+\kappa)}{k_{m} \kappa}
$$

and values of parameters $\alpha_{i}$ and $\gamma_{i}$ are given in Table 2. Whilst our focus here is on the qualitative nature of the fit, we note that the $R^{2}$ values obtained for the numerical fits shown in figure 5 are all above 0.983 .

In Figure 4(b), we indicate the accuracy of the linear approximation (4.11) in comparison to that provided by (4.13), highlighting that (4.11) provides a good approximation only for slow-moving waves. Figure 5 shows 
Table 2: Parameter values for boundary velocity estimate (4.13).

\begin{tabular}{cccc}
\hline Parameter & Value & Parameter & \multicolumn{1}{c}{ Value } \\
\hline$\alpha_{1}$ & -2.6 & $\gamma_{1}$ & $-2.8 \times 10^{-7}$ \\
$\alpha_{2}$ & -1.6 & $\gamma_{2}$ & $5.1 \times 10^{-6}$ \\
$\alpha_{3}$ & -0.49 & $\gamma_{3}$ & $-4.1 \times 10^{-5}$ \\
$\alpha_{4}$ & 0.53 & $\gamma_{4}$ & $1.0 \times 10^{-4}$ \\
\hline
\end{tabular}

the correspondence between the fitted function (4.13) and the numerical results.

In this section we have considered in detail the travelling wave speeds arising in the fully non-linear multiphase model that forms the basis of our macroscale description $(3.32,3.33,3.37,3.38)$, in order to inform its closure through constitutive specification of the boundary velocity $\boldsymbol{v}_{\Gamma}^{(1)} \cdot \boldsymbol{n}$. To facilitate numerical solution of this highly complex free-boundary problem, and to maintain consistency with the closely related study [41], we restrict attention to $1 \mathrm{D}$. There is, therefore, not a direct correspondence between the two models; the representation (4.13) nevertheless provides a useful approximation for $\boldsymbol{v}_{\Gamma}^{(1)} \cdot \boldsymbol{n}$ in (3.38), thereby enabling closure of our multiscale description.

\section{$5 \quad$ Numerical experiments}

We now present a series of illustrative numerical simulations of the microscopic cell problems and the corresponding effective macroscale description. The microscale problem (3.18-3.27) poses significant challenges since, unlike other studies of the type, the unit cell problems remain coupled to the macroscale dynamics. For simplicity, we therefore consider the case for which the water phase is inviscid and the more familiar micro-macro decoupling is achieved. First we recapitulate the relevant equations.

\subsection{Microscopic cell problem}

In this particular sub-case, we set $\mu=0$ (but retain the interphase viscous drag term). In this case, an appropriate ansatz is:

$$
\boldsymbol{v}_{i}^{(0)}=-\boldsymbol{K}_{i} \boldsymbol{\nabla}_{\boldsymbol{X}} p_{w}^{(0)} \text { and } p_{i}^{(1)}=-\boldsymbol{a}_{i} \boldsymbol{\nabla}_{\boldsymbol{X}} p_{w}^{(0)}-\bar{p}_{i} \text { for } i=n, w,
$$

where pressures $p_{w}^{(0)}, \bar{p}_{i}$ are microscale invariant and $\boldsymbol{K}_{i}, \boldsymbol{a}_{i}$ are tensors as described in section 3.3.1, with $\left\langle\boldsymbol{a}_{i}\right\rangle_{T}=\mathbf{0}$. In the tissue domain, $\Omega_{T}$, the 


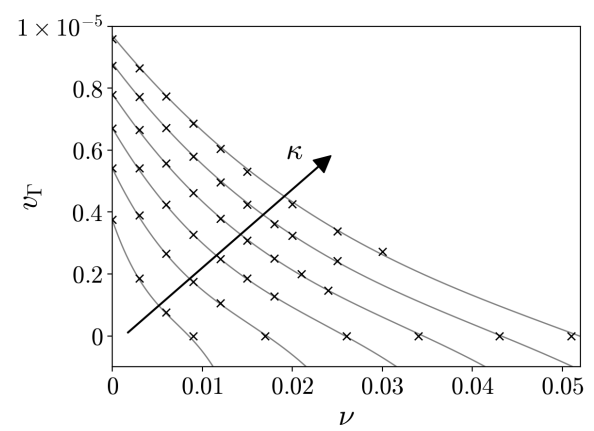

(a)

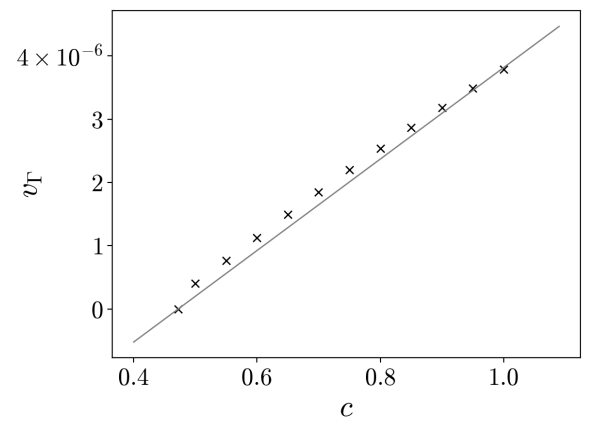

(c)

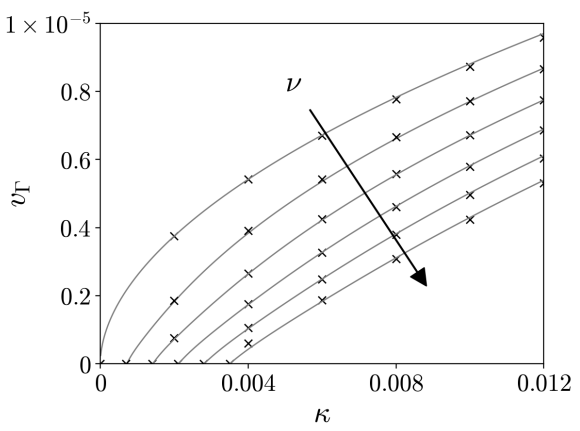

(b)

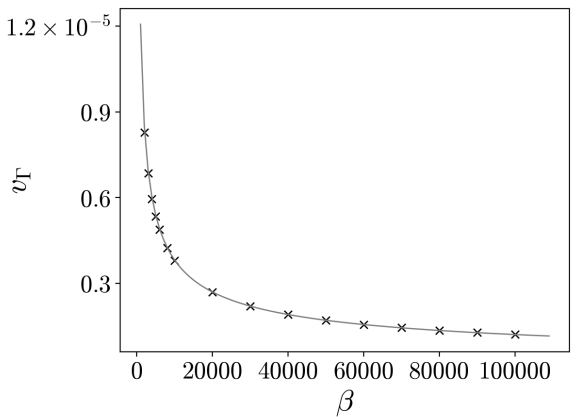

(d)

Figure 5: Graphs showing the relationship between boundary velocity of a forward travelling wave, found from the solution of (4.1) and (4.2), and (a) aggregation $\nu$, (b) repulsion $\kappa$, (c) nutrient concentration $c$ and (d) interphase $\operatorname{drag} \beta$. Crosses denote values obtained from numerics whilst solid lines are suggested fits to the data. Parameters (a) $\kappa=2 \times 10^{-3}$ $1.2 \times 10^{-2}$, (b) $\nu=0-1.2 \times 10^{-2}$. All other parameters as given in Table 1 . 
Stokes problem is given by:

$$
\begin{gathered}
\boldsymbol{\nabla}_{\boldsymbol{x}} \cdot \boldsymbol{K}_{n}^{\top}=\mathbf{0}, \quad \boldsymbol{\nabla}_{\boldsymbol{x}} \cdot \boldsymbol{K}_{w}^{\top}=\mathbf{0}, \\
\boldsymbol{\nabla}_{\boldsymbol{x}} \boldsymbol{a}_{w}^{\top}-\boldsymbol{I}-\beta \theta_{n}^{*}\left(\boldsymbol{K}_{n}-\boldsymbol{K}_{w}\right)=\mathbf{0}, \\
\boldsymbol{\nabla}_{\boldsymbol{x}} \boldsymbol{a}_{n}^{\top}-\left(1-\frac{1}{\theta_{n}^{*}}\right) \boldsymbol{I}-\nabla_{\boldsymbol{x}}^{2} \boldsymbol{K}_{n}-\beta \theta_{w}^{*}\left(\boldsymbol{K}_{w}-\boldsymbol{K}_{n}\right)=\mathbf{0} .
\end{gathered}
$$

On $\Gamma$ :

$$
\boldsymbol{K}_{n}^{\top} \boldsymbol{n}=\mathbf{0}, \quad \boldsymbol{K}_{w}^{\top} \boldsymbol{n}=\mathbf{0}, \quad\left(\theta_{n}^{*} \boldsymbol{K}_{n}^{\top}+\theta_{w}^{*} \boldsymbol{K}_{w}^{\top}\right) \boldsymbol{t}=\mathbf{0}
$$

On $\partial \Omega_{S}$ :

$$
\boldsymbol{K}_{n}=\mathbf{0}, \quad \boldsymbol{K}_{w}=\mathbf{0} .
$$

Finally solutions must be periodic on the boundaries of the periodic cell. The equations in this case have been simplified by the use of the continuity of pressures (3.10) which becomes $0=p_{w}^{(0)}+\theta_{n}^{*} \phi_{n}^{(0)}$.

In $\Omega_{I}$ equations (3.18-3.19) do not apply we, instead, have $p_{I}^{(0)}=0$ and, thereby, $\boldsymbol{v}_{I}=\mathbf{0}$. We note that despite the simplifications outlined above, the cell problem comprises a non-trivial coupled Darcy-Stokes problem. Together with its dependence on $\theta^{*}$ and the boundary conditions relevant to the microscale set-up it is therefore similar to, but distinct from, the cell problems encountered in other related studies [19].

\subsection{Macroscale description}

The macroscale description is given by $(3.32,3.33,3.37,3.38)$ where

$$
\begin{aligned}
& \overline{\boldsymbol{v}}^{(0)}=\left\langle\theta_{n}^{*} \boldsymbol{v}_{n}^{(0)}+\theta_{w}^{*} \boldsymbol{v}_{w}^{(0)}\right\rangle_{T} \\
& \overline{\boldsymbol{K}}=\left\langle\theta_{n}^{*} \boldsymbol{K}_{n}+\theta_{w}^{*} \boldsymbol{K}_{w}\right\rangle_{T}
\end{aligned}
$$

Additionally, we are required to specify the linearised growth and nutrient uptake functions for (3.37) and (3.38). For generality we choose $S_{n}^{(0)}$ and $\Lambda^{(0)}$ that support non-trivial steady states $\theta_{n}^{*}, c^{*}$ as follows:

$$
\begin{aligned}
S_{n}^{(0)} & = \begin{cases}k_{m} \theta_{n}^{*}\left(1-\theta_{n}^{*}\right) c^{(0)} & \text { if } c^{(0)}>0, \\
k_{n} \theta_{n}^{*} c^{(0)} & \text { if } c^{(0)}<0,\end{cases} \\
\Lambda^{(0)} & = \begin{cases}\lambda k_{m} \theta_{n}^{*} c^{(0)} & \text { if } c^{(0)}>0, \\
0 & \text { if } c^{(0)}<0 .\end{cases}
\end{aligned}
$$

Such a choice corresponds to a threshold (steady state) nutrient concentration, $c^{*}$, below which cell growth is negligible whilst above it we neglect cell death. Finally, we take $\boldsymbol{v}_{\Gamma}^{(1)} \cdot \boldsymbol{n}$ to be some constant multiple of (4.13), with this additional parameter denoted $\Upsilon$. 


\subsection{Microscale Problem}

We now present representative numerical experiments demonstrating the numerical approximation to the solution of the microscale system derived in section 3.3.1. Our focus here is to parameterize the dependence of macroscopic model derived in section 3.3.2 on the underlying microscopic geometry. For computational simplicity, we consider only two-dimensional calculations. However, we note that the methods employed here translate naturally to three dimensions.

In the following computations we consider the microscale geometry $\Omega=$ $(0,1)^{2}$, decomposed into disjoint subsets $\Omega_{I}, \Omega_{T}$ and $\Omega_{S}$. For simplicity, we choose a symmetric geometry that may be parameterized by five parameters $\left(r_{E}, r_{I}, r_{C}, \ell_{1}\right.$ and $\left.\ell_{2}\right)$. A schematic diagram of the microscale geometry is provided in Figure 6.

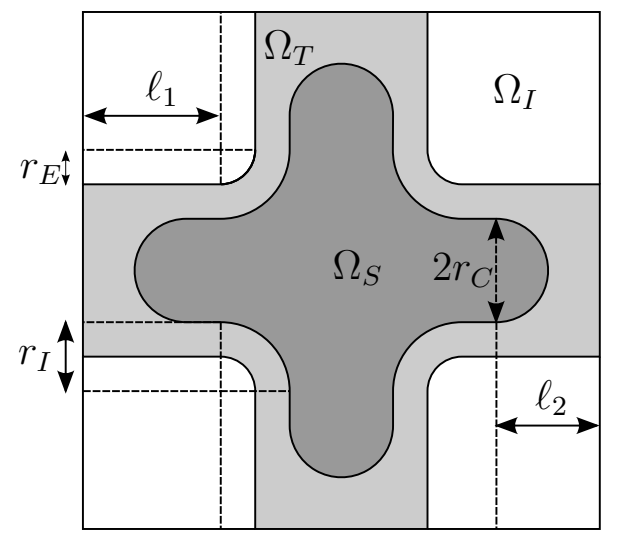

Figure 6: Schematic diagram of the microscale domain, $\Omega$, employed in the example numerical solution of equations (5.2-5.4).

It is beyond the scope of this work to provide a full description of the discretizations applied to the microscale PDEs; here we provide only details of the finite element spaces and application of boundary conditions and appropriate references. We employ standard finite element methods for the coupled microscale system, approximating $\left(\boldsymbol{K}_{w}, \boldsymbol{a}_{w}\right)$ with mixed Raviart-Thomas/discontinuous Galerkin elements [11, 64], and $\left(\boldsymbol{K}_{n}, \boldsymbol{a}_{n}\right)$ with Taylor-Hood elements [72]. The periodic boundary conditions on $\partial \Omega_{T} \cap \partial \Omega$ are strongly imposed on the approximation of $\boldsymbol{K}_{w}$ and $\boldsymbol{K}_{n}$. Similarly, the boundary conditions for $\boldsymbol{K}_{w}$ on $\Gamma$ given by (5.5), and both $\boldsymbol{K}_{n}$ and $\boldsymbol{K}_{w}$ on $\partial \Omega_{S}$ given by (5.6) are strongly imposed. Finally, the condition on $\boldsymbol{K}_{n}$ on $\Gamma$ given by (5.5) is weakly imposed, see [4, 51]. Figure 7 shows representative numerical solutions of the microscale system obtained using the parameter values provided in Table 3. Further, we note that under the assumptions on the geometrical parameters, the sizes of the respective domains are given by $\left|\Omega_{I}\right|=0.36,\left|\Omega_{T}\right|=0.48$ and $\left|\Omega_{S}\right|=0.16$ (i.e. the 
porosity is $84 \%$, in line with [29]).

Table 3: Parameter values employed in the microscale numerical experiment.

\begin{tabular}{cc}
\hline Parameter & Value \\
\hline$\beta$ & $1.0 \times 10^{4}$ \\
$\theta_{n}^{*}$ & 0.8111 \\
$\theta_{w}^{*}$ & 0.1889 \\
$r_{E}$ & 0.05 \\
$r_{I}$ & 0.2 \\
$r_{C}$ & 0.05 \\
$\ell_{1}$ & 0.25 \\
$\ell_{2}$ & 0.2 \\
\hline
\end{tabular}

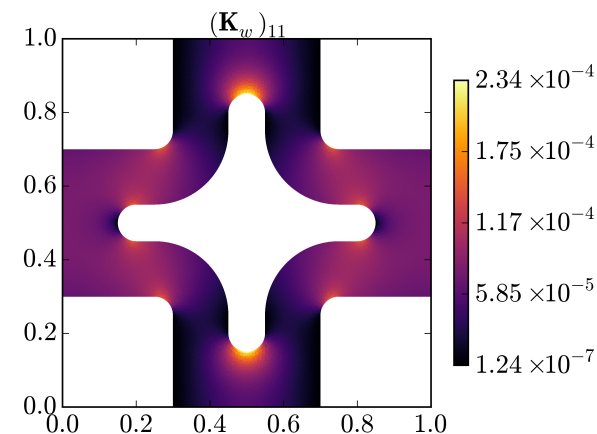

(a)

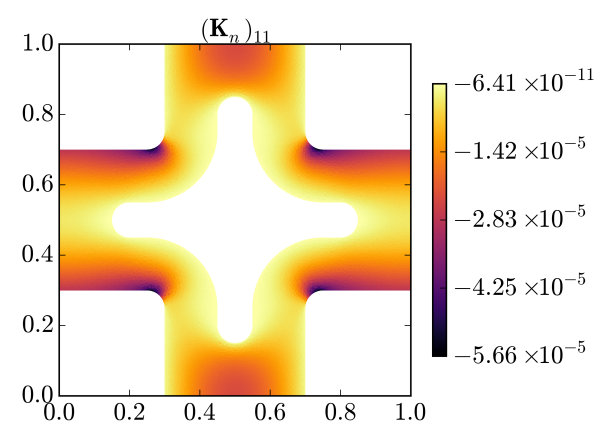

(c)

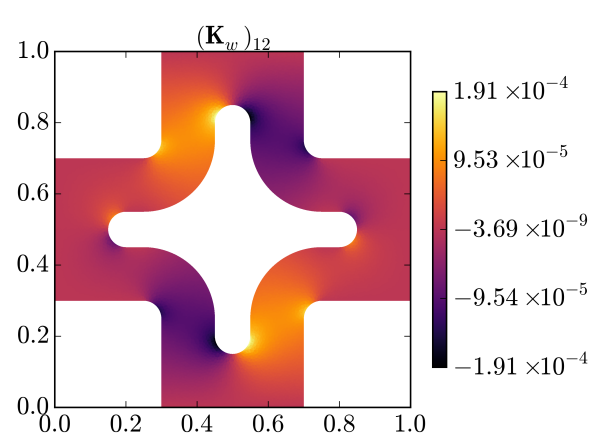

(b)

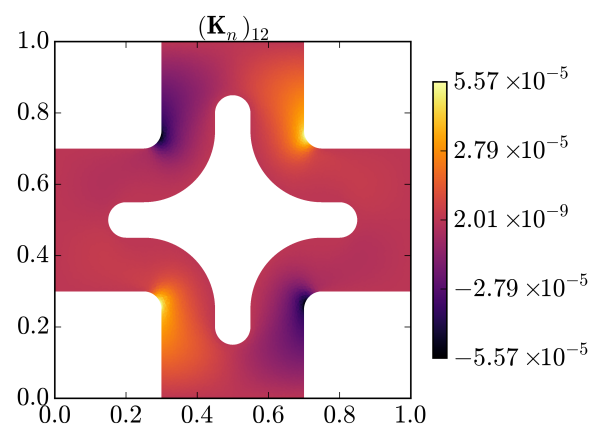

(d)

Figure 7: Numerical approximation of $\boldsymbol{K}_{n}$ and $\boldsymbol{K}_{w}$, from the solution of the microscale problem given by equations $(5.2-5.4)$.

Due to the symmetries of $\Omega_{I}, \Omega_{T}$ and $\Omega_{S}$, the computation of $\left(\boldsymbol{K}_{n / w}\right)_{11}$ and $\left(\boldsymbol{K}_{n / w}\right)_{12}$ is sufficient to specify $\boldsymbol{K}_{n / w}$ in $\Omega_{T}$. Given this observation, we may use the numerical approximations shown in Figure 7 to calculate 
the spatially averaged permeabilities as

$$
\left\langle\boldsymbol{K}_{w}\right\rangle_{T}=\left(\begin{array}{cc}
3.01 \times 10^{-4} & -5.81 \times 10^{-11} \\
-5.81 \times 10^{-11} & 3.01 \times 10^{-4}
\end{array}\right)
$$

and

$$
\left\langle\boldsymbol{K}_{n}\right\rangle_{T}=\left(\begin{array}{ll}
-5.16 \times 10^{-6} & -2.90 \times 10^{-11} \\
-2.90 \times 10^{-11} & -5.16 \times 10^{-6}
\end{array}\right) .
$$

We note that the off-diagonal entries of $\left\langle\boldsymbol{K}_{w}\right\rangle_{T}$ and $\left\langle\boldsymbol{K}_{n}\right\rangle_{T}$ are, effectively, zero: under the choice of geometry shown in Figure 6, we would expect $\left\langle\boldsymbol{K}_{w}\right\rangle_{T}$ and $\left\langle\boldsymbol{K}_{n}\right\rangle_{T}$ to be isotropic, to within the discretization error in numerical scheme, and the results (5.11) and (5.12) are consistent with this.

\subsection{Macroscale Problem}

We now consider numerical experiments demonstrating the corresponding macroscopic behaviour of the system derived in section 3.3.2. We highlight that our focus here is to highlight the macroscale model dynamics that arise from a suitable consideration of the multiscale growth processes together with appropriate numerical methods to facilitate their calculation. Representative parameter values, boundary and initial data, and geometry are considered, in conjunction with microscale data obtained from the numerical experiments discussed in section 5.3. For computational simplicity, we once more restrict our attention to twodimensional calculations, though we again note that the methods employed here generalise naturally.

Denoting the macroscale domain and its boundary, by $\Omega_{L}$ and $\partial \Omega_{L}$, respectively, we specify the pressure boundary condition as

$$
p_{w}^{(0)}=0 \quad \text { on } \partial \Omega_{L},
$$

and initial conditions, at $t=0$,

$$
c^{(0)}=1 \quad \text { in } \Omega_{L}
$$

and

$$
\theta_{n}^{(0)}=0.6 \quad \text { in } \Omega_{L},
$$

on the nutrient and cell volume fractions, respectively. Further, we define our macroscale domain to be $\Omega_{L}=\left\{\boldsymbol{X}: \sqrt{X_{1}^{2}+X_{2}^{2}}<1\right\}$.

To compute the numerical approximation of the macroscale flow system we employ a mixed Raviart-Thomas/discontinuous Galerkin finite element $[11,64]$. As there is no spatial component to the evolution of the cell 
volume fraction and nutrient concentration, we simply employ an explicit Euler time-stepping scheme in the discretization of (3.38) and (3.37).

Figure 8 shows the dependence of $\theta_{n}^{(0)}$ on a range of parameters in the macroscale model. The effect of cell-cell aggregation and repulsion on the cell volume fraction is illustrated by Figures 8(a) and (b), showing that increased repulsion or decreased aggregation results in lower $\theta_{n}^{(0)}$. Figures $8(\mathrm{c})$ and $(\mathrm{d})$ show the dependence of $\theta_{n}^{(0)}$ and $c^{(0)}$ on $\lambda$, with increasing uptake depleting nutrient faster and hence leading to slower increase in $\theta_{n}^{(0)}$. Figure $8(\mathrm{e})$ depicts the effect of varying $\Upsilon$ on $\theta_{n}^{(0)}$. We note that $c^{(0)}$ does not vary strongly with other parameters in the model, and hence forgo the inclusion of those plots here. In the numerical results presented in this figure we consider only the case $\bar{\rho}=1$. As there is no density difference, however, the forcing in the flow equations reduces to zero and the pressure $p_{w}^{(0)}$ is identically zero throughout $\Omega_{L}$.

In the case where there is a moderate density difference between the viscous and inviscid fluids we obtain a non-trivial pressure $p_{w}^{(0)}$, the evolution of which is shown in Figure 9. At this level of density difference, however, (and for the other parameter values chosen) the differences in evolution of both $\theta_{n}^{(0)}$ and $c^{(0)}$ are small and, therefore, we do not include those figures here. Over the course of the simulation we are able to observe an initial increase in the pressure inside the domain, as the cell volume fraction decreases (cf. Figure 8(e)), followed by a decrease in pressure as the cell volume fraction increases.

\section{Discussion}

We have derived an effective macroscale description for tissue growth, from an underlying microscale model, using multiscale homogenisation. We considered a rigid periodic lattice-like structure covered by a layer of growing tissue. The model is therefore applicable to problems in regenerative medicine, such as tissue growth within a tissue engineering scaffold (our primary motivation), or biofilm growth, for example in the subsurface or the fouling of filters. The tissue, modelled at the microscale as a two phase mixture, consists of cells and culture medium (water), both modelled as viscous fluids. The remaining pore space contains water only.

The method of multiscale homogenisation has been used previously for biologically-inspired models, including fluid and nutrient or drug transport [69] and, more recently, in studies that additionally incorporate growth $[17,19,54,61]$. Here, rather than a solid tissue undergoing surface [54, 61] or volumetric growth [17], we considered a multiphase fluid tissue. This approach was used in [19] with strong interphase drag imposed, resulting in equal phase velocities and growth only occurring in a thin boundary 


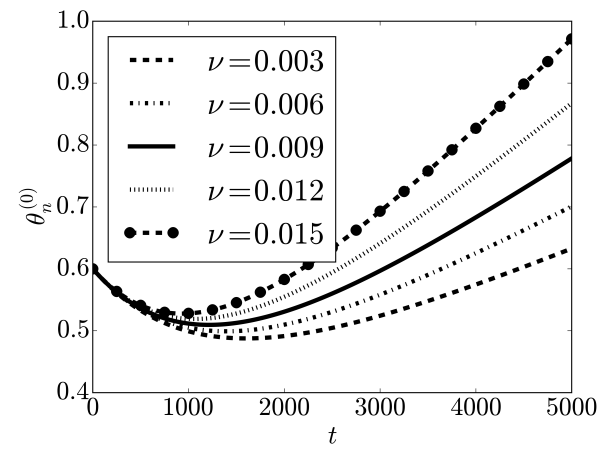

(a) Dependence of $\theta_{n}^{(0)}$ on $\nu$ with $\Upsilon=0.05, \lambda=10, \kappa=0.012$.

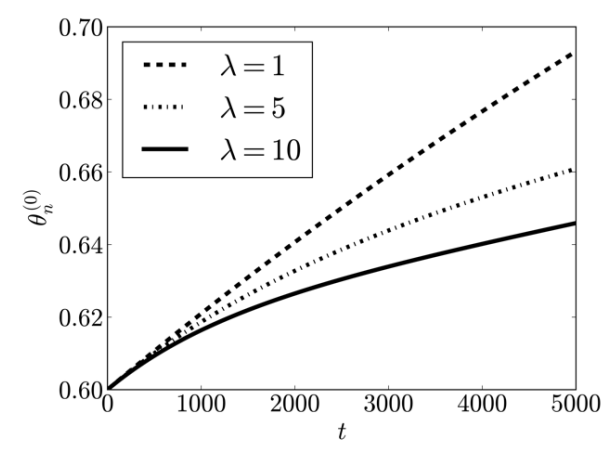

(c) Dependence of $\theta_{n}^{(0)}$ on $\lambda$ with $\Upsilon=0.001, \nu=0.009, \kappa=0.006$.

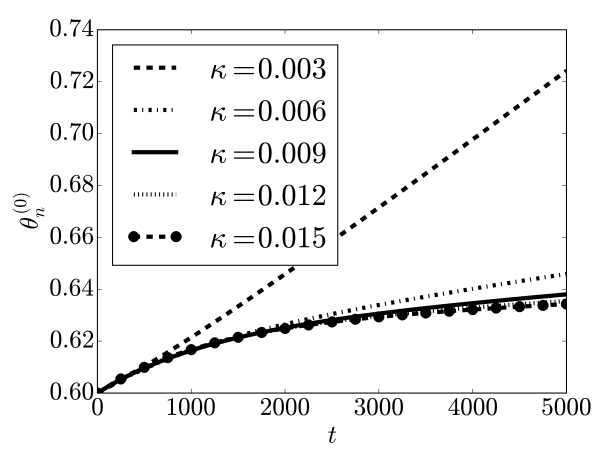

(b) Dependence of $\theta_{n}^{(0)}$ on $\kappa$ with $\Upsilon=0.001, \nu=0.009, \lambda=10$.

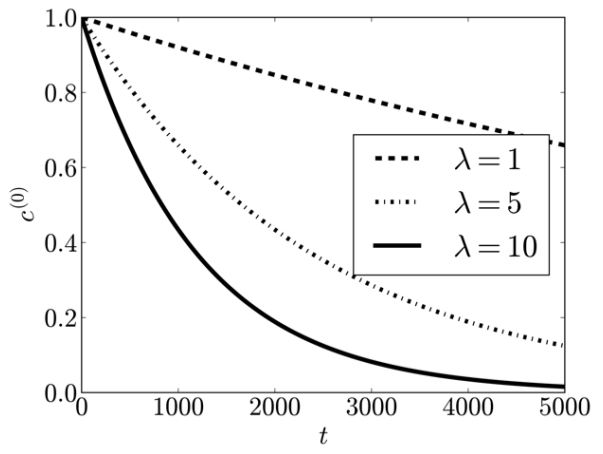

(d) Dependence of $c^{(0)}$ on $\lambda$ with $\Upsilon=0.001, \nu=0.009, \kappa=0.006$.

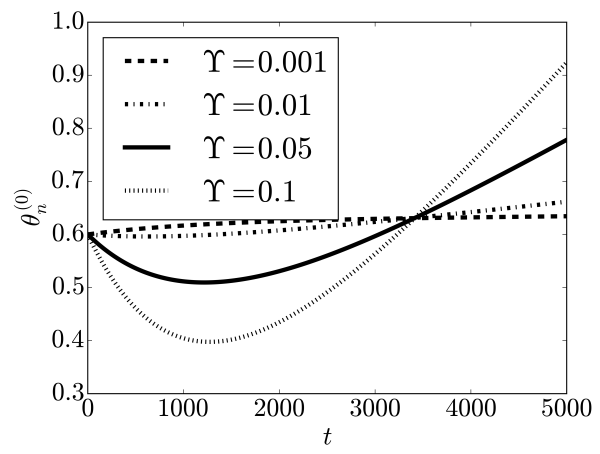

(e) Dependence of $\theta_{n}^{(0)}$ on $\Upsilon$ with $\kappa=0.006, \nu=0.009, \lambda=10$.

Figure 8: Plots demonstrating the dependence of the evolution of nutrient concentration and cell volume fraction on parameters in the macroscale model, described by equations $(3.32,3.33,3.37,3.38)$ and boundary conditions $(5.13-5.15)$ with $\bar{\rho}=1$.

layer. To permit active cell motion and therefore allow a more complex description of tissue growth, we relaxed this assumption. A fully nonlinear 


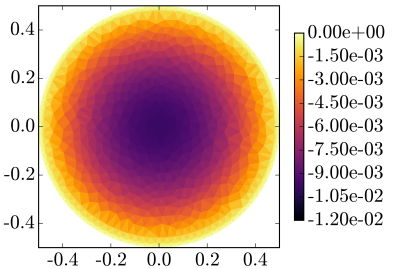

(a) $t=0$

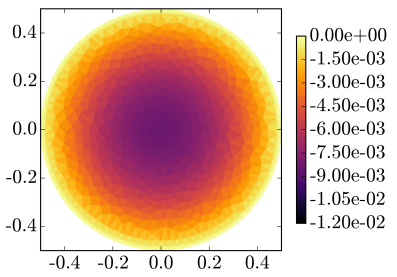

(d) $t=1500$

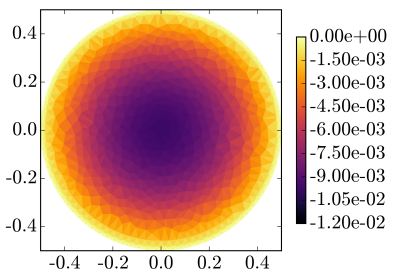

(g) $t=3000$

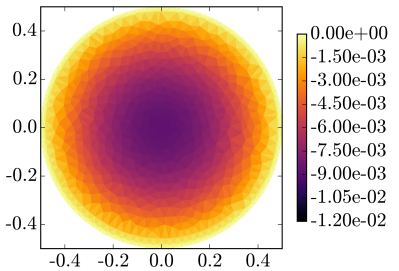

(b) $t=500$

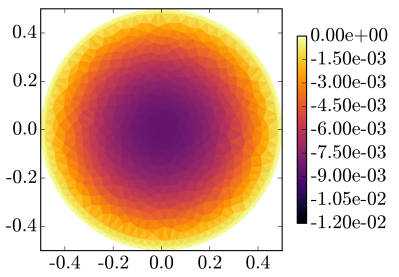

(e) $t=2000$

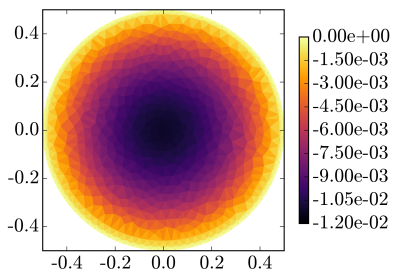

(h) $t=4000$

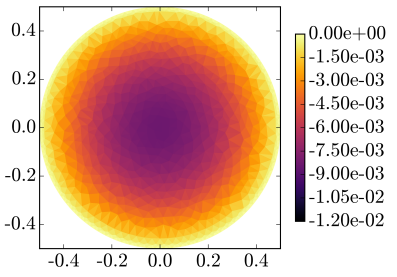

(c) $t=1000$

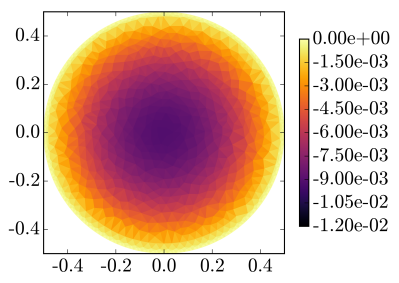

(f) $t=2500$

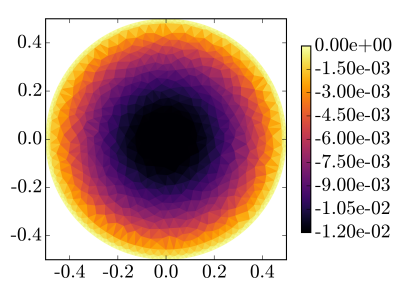

(i) $t=5000$

Figure 9: Plots showing the leading order pressure for $\Upsilon=0.05, \nu=0.009$, $\kappa=0.012, \bar{\rho}=1.5$, and $\lambda=10$, from the solution of the macroscale problem described by equations $(3.32,3.33,3.37,3.38)$ and boundary conditions $(5.13-$ $5.15)$.

analysis of the type considered in [17] is complicated by the interphase mass transfer that we accommodate. Moreover, since we seek a tractable description, we consider a linearisation of the model that permits a more straightforward multiscale analysis. Despite this, in the general case, the resulting formulation nevertheless presents significant challenges due to the coupling between micro- and macro-scale dynamics.

We obtain an effective description of tissue growth that comprises a Darcy flow, dependent on the microstructure, and partial differential equations for the volume fraction of cells within the scaffold and the concentration of some generic nutrient, required for growth. This macroscale formulation is parameterised by permeability tensors, with multiscale dependence, provided by a set of modified Stokes-type cell problems. As is a common feature with this kind of analysis, we do not have sufficient information to obtain a closed macroscale model. A constitutive statement for the microscale motion of the free tissue boundary is required, 
which we motivate by considering the travelling wave behaviour of a closely related one dimensional model, obtaining a polynomial relationship between the boundary velocity and the model parameters.

Due to the challenges associated with the coupling of the unit cell problem to the macroscale dynamics, in our illustrative numerical experiments we focussed on the case where the water phase is inviscid. In particular, we consider a representative two dimensional geometry and show how the solution to the relevant cell problems parameterises the corresponding macroscale description. The dependence of macroscale cell volume fraction on a range of parameters, cell aggregation, repulsion, boundary velocity and nutrient uptake, over time is illustrated. For moderately different phase densities the behaviour of macroscale pressure is shown, whilst a lack of density difference results in trivial pressure.

In the example simulations we have considered a problem for which there is no macroscale dependence in the cell problem. A significant extension to this would be to consider numerical examples for the viscousviscous case, in which there is explicit macroscale dependence in the cell problem. As the macroscopic variables are constant on the cell (as a result of the strong separation of lengthscales), we are able to view the cell problems as high-dimensional parametric PDEs. As such, there are possible means of reducing computational requirements though use of techniques such as empirical interpolation [3], or decomposition methods such as proper orthogonal decomposition (POD) and dynamic mode decomposition (DMD) [28, 49, 68].

Another important extension of the model would be to better understand the backward travelling wave behaviour and hence deduce a suitable expression for $\boldsymbol{v}_{\Gamma}^{(1)} \cdot \boldsymbol{n}$ in this case. This would allow nutrient deficiency, an important consideration in the growth of tissue, to be investigated using the effective macroscale description derived. Additionally, further investigation into the connection between the constitutive choices employed in the multiphase model employed and the functional form of the tissue-interstitium boundary velocity at a deeper level would be of general interest and would greatly enhance the impact of the current work.

There are limitations to the model. In particular we note that the linearised equations describe the dynamics close to the steady state, which itself is severely limited by the assumptions made, therefore, the extension to the fully nonlinear case forms important future work. 


\section{Acknowledgement}

JC acknowledges the support of Engineering and Physical Sciences Research Council grant number EP/K039342/1. 


\section{References}

[1] S. Astanin and L. Preziosi. Multiphase models of tumour growth. In Selected topics in cancer modeling, pages 1-31. Springer, 2008.

[2] L. Band and J. King. Multiscale modelling of auxin transport in the plant-root elongation zone. Journal of mathematical biology, 65(4):743-785, 2012.

[3] M. Barrault, Y. Maday, N. C. Nguyen, and A. T. Patera. An 'empirical interpolation'method: application to efficient reducedbasis discretization of partial differential equations. Comptes Rendus Mathematique, 339(9):667-672, 2004.

[4] Y. Bazilevs and T. J. R. Hughes. Weak imposition of Dirichlet boundary conditions in fluid mechanics. Computers \& Fluids, 36(1):12-26, 2007. Challenges and Advances in Flow Simulation and Modeling.

[5] A. Bensoussan, J.-L. Lions, and G. Papanicolaou. Asymptotic analysis for periodic structures, volume 5. North-Holland Publishing Company Amsterdam, 1978.

[6] T. Berman, R. Mizrahi, and C. G. Dosoretz. Transparent exopolymer particles (tep): A critical factor in aquatic biofilm initiation and fouling on filtration membranes. Desalination, 276(1):184-190, 2011.

[7] Z. F. Bhat and H. Fayaz. Prospectus of cultured meat-advancing meat alternatives. Journal of Food Science and Technology, 48(2):125$140,2011$.

[8] L. Bowden, P. Maini, D. Moulton, J. Tang, X. Wang, P. Liu, and H. Byrne. An ordinary differential equation model for full thickness wounds and the effects of diabetes. Journal of Theoretical Biology, 361:87-100, 2014.

[9] R. M. Bowen. Incompressible porous media models by use of the theory of mixtures. International Journal of Engineering Science, 18(9):11291148, 1980.

[10] C. Breward, H. Byrne, and C. Lewis. The role of cell-cell interactions in a two-phase model for avascular tumour growth. Journal of Mathematical Biology, 45(2):125-152, 2002.

[11] F. Brezzi and M. Fortin. Mixed and Hybrid Finite Element Methods. Springer-Verlag, New York, 1991. 
[12] D. L. Brown, P. Popov, and Y. Efendiev. Effective equations for fluidstructure interaction with applications to poroelasticity. Applicable Analysis, 93(4):771-790, 2014.

[13] R. Burridge and J. B. Keller. Poroelasticity equations derived from microstructure. The Journal of the Acoustical Society of America, 70(4):1140-1146, 1981.

[14] H. Byrne and L. Preziosi. Modelling solid tumour growth using the theory of mixtures. Mathematical Medicine and Biology, 20(4):341366, 2003.

[15] H. M. Byrne, J. R. King, D. S. McElwain, and L. Preziosi. A twophase model of solid tumour growth. Applied Mathematics Letters, 16(4):567-573, 2003.

[16] Y. Chen, S. Zhou, and Q. Li. Microstructure design of biodegradable scaffold and its effect on tissue regeneration. Biomaterials, 32(22):5003-5014, 2011.

[17] J. Collis, D. Brown, M. E. Hubbard, and R. O'Dea. Effective equations governing an active poroelastic medium. In Proceedings of the Royal Society A., volume 473. The Royal Society, 2017.

[18] J. Collis, M. E. Hubbard, and R. D. O'Dea. Computational modelling of multiscale, multiphase fluid mixtures with application to tumour growth. Computer Methods in Applied Mechanics and Engineering, 309:554-578, 2016.

[19] J. Collis, M. E. Hubbard, and R. D. O'Dea. A multi-scale analysis of drug transport and response for a multi-phase tumour model. European Journal of Applied Mathematics, pages 1-36, 2016.

[20] P. Coombs, D. Wagner, K. Bateman, H. Harrison, A. Milodowski, D. Noy, and J. West. The role of biofilms in subsurface transport processes. Quarterly Journal of Engineering Geology and Hydrogeology, 43(2):131-139, 2010.

[21] V. Cristini, X. Li, J. S. Lowengrub, and S. M. Wise. Nonlinear simulations of solid tumor growth using a mixture model: invasion and branching. Journal of Mathematical Biology, 58(4):723-763, 2009.

[22] Y. Davit, C. G. Bell, H. Byrne, L. A. Chapman, L. S. Kimpton, G. E. Lang, K. H. Leonard, J. M. Oliver, N. Pearson, R. Shipley, et al. Homogenization via formal multiscale asymptotics and volume averaging: How do the two techniques compare? Advances in Water Resources, 62:178-206, 2013. 
[23] D. A. Drew. Averaged field equations for two-phase media. Studies in Applied Mathematics, 50(2):133-166, 1971.

[24] D. A. Drew and S. L. Passman. Theory of multicomponent fluids, volume 135. Springer Science \& Business Media, 2006.

[25] A. El-Armouche, J. Singh, H. Naito, K. Wittköpper, M. Didié, A. Laatsch, W.-H. Zimmermann, and T. Eschenhagen. Adenovirusdelivered short hairpin RNA targeting PKC $\alpha$ improves contractile function in reconstituted heart tissue. Journal of Molecular and Cellular Cardiology, 43(3):371-376, 2007.

[26] S. Ferguson, J. Bryant, R. Ganz, and K. Ito. The influence of the acetabular labrum on hip joint cartilage consolidation: a poroelastic finite element model. Journal of Biomechanics, 33(8):953-960, 2000.

[27] S. Franks and J. King. Interactions between a uniformly proliferating tumour and its surroundings: uniform material properties. Mathematical Medicine and Biology, 20(1):47-89, 2003.

[28] M. Ghommem, M. Presho, V. M. Calo, and Y. Efendiev. Mode decomposition methods for flows in high-contrast porous media: Global-local approach. Journal of Computational Physics, 253:226 238, 2013.

[29] S. Grenier, M. Sandig, D. W. Holdsworth, and K. Mequanint. Interactions of coronary artery smooth muscle cells with $3 \mathrm{~d}$ porous polyurethane scaffolds. Journal of Biomedical Materials Research Part A, 89(2):293-303, 2009.

[30] U. Hornung. Homogenization and porous media, volume 6. Springer Science \& Business Media, 2012.

[31] M. Hubbard and H. Byrne. Multiphase modelling of vascular tumour growth in two spatial dimensions. Journal of Theoretical Biology, 316:70-89, 2013.

[32] M. E. Hubbard and N. Dodd. A 2d numerical model of wave run-up and overtopping. Coastal Engineering, 47(1):1-26, 2002.

[33] Y. Ichikawa, K. Kawamura, N. Fujii, and T. Nattavut. Molecular dynamics and multiscale homogenization analysis of seepage/diffusion problem in bentonite clay. International Journal for Numerical Methods in Engineering, 54(12):1717-1749, 2002.

[34] L. Irons, J. Collis, and R. O'Dea. Microscale Transport Modelling in Biological Processes, chapter Microstructural influences on growth and transport in biological tissue: a multiscale description. Elsevier, 2017. 
[35] R. K. Jain. Delivery of molecular and cellular medicine to solid tumors. Advanced Drug Delivery Reviews, 46(1):149-168, 2001.

[36] A. E. Jakus, E. B. Secor, A. L. Rutz, S. W. Jordan, M. C. Hersam, and R. N. Shah. Three-dimensional printing of high-content graphene scaffolds for electronic and biomedical applications. ACS Nano, 9(4):4636-4648, 2015.

[37] J. B. Keller. Effective behavior of heterogeneous media. In Statistical mechanics and statistical methods in theory and application, pages 631-644. Springer, 1977.

[38] R. K. Korhonen, M. S. Laasanen, J. Töyräs, R. Lappalainen, H. J. Helminen, and J. S. Jurvelin. Fibril reinforced poroelastic model predicts specifically mechanical behavior of normal, proteoglycan depleted and collagen degraded articular cartilage. Journal of Biomechanics, 36(9):1373-1379, 2003.

[39] E. W. Larsen and J. B. Keller. Asymptotic solution of neutron transport problems for small mean free paths. Journal of Mathematical Physics, 15(1):75-81, 1974.

[40] G. Lemon and J. King. Multiphase modelling of cell behaviour on artificial scaffolds: effects of nutrient depletion and spatially nonuniform porosity. Mathematical Medicine and Biology, 24(1):5783, 2007.

[41] G. Lemon and J. King. Travelling-wave behaviour in a multiphase model of a population of cells in an artificial scaffold. Journal of Mathematical Biology, 55(4):449-480, 2007.

[42] G. Lemon, J. King, H. Byrne, O. Jensen, and K. Shakesheff. Mathematical modelling of engineered tissue growth using a multiphase porous flow mixture theory. Journal of Mathematical Biology, 52(5):571-594, 2006.

[43] L. Li, J. Soulhat, M. Buschmann, and A. Shirazi-Adl. Nonlinear analysis of cartilage in unconfined ramp compression using a fibril reinforced poroelastic model. Clinical Biomechanics, 14(9):673-682, 1999.

[44] C. Y. Lin, N. Kikuchi, and S. J. Hollister. A novel method for biomaterial scaffold internal architecture design to match bone elastic properties with desired porosity. Journal of Biomechanics, 37(5):623636, 2004. 
[45] S. Lubkin and T. Jackson. Multiphase mechanics of capsule formation in tumors. Transactions-American Society of Mechanical Engineers Journal of Biomechanical Engineering, 124(2):237-243, 2002.

[46] A. Mak. The apparent viscoelastic behavior of articular cartilage - the contributions from the intrinsic matrix viscoelasticity and interstitial fluid flows. Journal of Biomechanical Engineering, 108(2):123-130, 1986.

[47] C. Marle. On macroscopic equations governing multiphase flow with diffusion and chemical reactions in porous media. International Journal of Engineering Science, 20(5):643-662, 1982.

[48] A. Matzavinos and M. Ptashnyk. Homogenization of oxygen transport in biological tissues. Applicable Analysis, 95(5):1013-1049, 2016.

[49] G. Mehdi, V. M. Calo, and Y. Efendiev. Mode decomposition methods for flows in high-contrast porous media. a global approach. Journal of Computational Physics, 257, Part A:400 - 413, 2014.

[50] C. C. Mei and B. Vernescu. Homogenization methods for multiscale mechanics. World scientific, 2010.

[51] J. A. Nitsche. Über ein variationsprinzip zur lösung dirichlet-problemen bei verwendung von teilräumen die keinen randbedingungen unteworfen sind. Abh. Math. Sem. Univ. Hamburg, $36: 9-15,1971$.

[52] R. O'Dea, H. Byrne, and S. Waters. Continuum modelling of in vitro tissue engineering: a review. In Computational Modeling in Tissue Engineering, pages 229-266. Springer, 2013.

[53] R. O'Dea and J. King. Multiscale analysis of pattern formation via intercellular signalling. Mathematical Biosciences, 231(2):172-185, 2011.

[54] R. O'Dea, M. Nelson, A. El Haj, S. Waters, and H. Byrne. A multiscale analysis of nutrient transport and biological tissue growth in vitro. Mathematical Medicine and Biology, 32(3):345-366, 2014.

[55] R. O'Dea, S. Waters, and H. Byrne. A multiphase model for tissue construct growth in a perfusion bioreactor. Mathematical Medicine and Biology, 27(2):95-127, 2010.

[56] R. D. O'Dea, S. L. Waters, and H. M. Byrne. A two-fluid model for tissue growth within a dynamic flow environment. European Journal of Applied Mathematics, 19(06):607-634, 2008. 
[57] J. Osborne, R. D. O’Dea, J. Whiteley, H. Byrne, and S. Waters. The influence of bioreactor geometry and the mechanical environment on engineered tissues. Journal of Biomechanical Engineering, 132(5):051006, 2010.

[58] M. Owen, T. Alarcón, P. Maini, and H. Byrne. Angiogenesis and vascular remodelling in normal and cancerous tissues. Journal of Mathematical Biology, 58(4-5):689-721, 2009.

[59] G. A. Pavliotis and A. Stuart. Multiscale methods: averaging and homogenization. Springer Science \& Business Media, 2008.

[60] N. Pearson, R. Shipley, S. Waters, and J. Oliver. Multiphase modelling of the influence of fluid flow and chemical concentration on tissue growth in a hollow fibre membrane bioreactor. Mathematical Medicine and Biology, 31(4):393-430, 2014.

[61] R. Penta, D. Ambrosi, and R. Shipley. Effective governing equations for poroelastic growing media. The Quarterly Journal of Mechanics and Applied Mathematics, 67(1):69-91, 2014.

[62] P. Popov, Y. Efendiev, and G. Qin. Multiscale modeling and simulations of flows in naturally fractured karst reservoirs. Communications in Computational Physics, 6(1):162, 2009.

[63] M. Ptashnyk and T. Roose. Derivation of a macroscopic model for transport of strongly sorbed solutes in the soil using homogenization theory. SIAM Journal on Applied Mathematics, 70(7):2097-2118, 2010 .

[64] P. A. Raviart and J. M. Thomas. A mixed finite element method for second order elliptic problems. In I. Galligani and E. Magenes, editors, Mathematical Aspects of the Finite Element Method, Lectures Notes in Math. 606. Springer-Verlag, New York, 1977.

[65] C. Rosenberg. Wound healing in the patient with diabetes mellitus. The Nursing clinics of North America, 25(1):247-261, 1990.

[66] E. Sánchez-Palencia. Non-homogeneous media and vibration theory. In Non-homogeneous media and vibration theory, volume 127, 1980.

[67] S. Schaaf, A. Shibamiya, M. Mewe, A. Eder, A. Stöhr, M. N. Hirt, T. Rau, W.-H. Zimmermann, L. Conradi, T. Eschenhagen, et al. Human engineered heart tissue as a versatile tool in basic research and preclinical toxicology. PloS one, 6(10):e26397, 2011. 
[68] P. J. Schmid. Dynamic mode decomposition of numerical and experimental data. Journal of Fluid Mechanics, 656:5-28, 2010.

[69] R. Shipley and S. Chapman. Multiscale modelling of fluid and drug transport in vascular tumours. Bulletin of Mathematical Biology, 72(6):1464-1491, 2010.

[70] R. Shipley, G. Jones, R. Dyson, B. Sengers, C. Bailey, C. Catt, C. Please, and J. Malda. Design criteria for a printed tissue engineering construct: a mathematical homogenization approach. Journal of Theoretical Biology, 259(3):489-502, 2009.

[71] J. J. Song, J. P. Guyette, S. E. Gilpin, G. Gonzalez, J. P. Vacanti, and H. C. Ott. Regeneration and experimental orthotopic transplantation of a bioengineered kidney. Nature Medicine, 19(5):646-651, 2013.

[72] C. Taylor and P. Hood. A numerical solution of the navier-stokes equations using the finite element technique. Computers $\&$ Fluids, 1(1):73 - 100, 1973.

[73] C. Truesdell. Rational thermodynamics. Springer Science \& Business Media, 2012.

[74] B. Van Leer. Towards the ultimate conservative difference scheme. V. A second-order sequel to Godunov's method. Journal of Computational Physics, 32(1):101-136, 1979.

[75] J. Visser, F. P. Melchels, J. E. Jeon, E. M. van Bussel, L. S. Kimpton, H. M. Byrne, W. J. Dhert, P. D. Dalton, D. W. Hutmacher, and J. Malda. Reinforcement of hydrogels using three-dimensionally printed microfibres. Nature communications, 6, 2015.

[76] J. Ward and J. King. Mathematical modelling of avascular-tumour growth ii: modelling growth saturation. Mathematical Medicine and Biology, 16(2):171-211, 1999.

[77] J. P. Ward and J. King. Mathematical modelling of avascular-tumour growth. Mathematical Medicine and Biology, 14(1):39-69, 1997.

[78] S. Waters, L. Cummings, K. Shakesheff, and F. Rose. Tissue growth in a rotating bioreactor. part i: mechanical stability. Mathematical Medicine and Biology, 23(4):311-337, 2006.

[79] C. Wei and J. Dong. Direct fabrication of high-resolution three-dimensional polymeric scaffolds using electrohydrodynamic hot jet plotting. Journal of Micromechanics and Microengineering, 23(2):025017, 2013. 